\title{
Pre-chemotherapy differences in visuospatial working memory in breast cancer patients compared to controls: an fMRI study
}

\author{
Carole Scherling $^{1}{ }^{*}$, Barbara Collins ${ }^{1,2}$, Joyce MacKenzie ${ }^{2}$, Catherine Bielajew $^{1}$ and Andra Smith ${ }^{1}$ \\ School of Psychology, University of Ottawa, Ottawa, ON, Canada \\ 2 Ottawa Hospital, Ottawa, ON, Canada
}

Edited by:

Srikantan S. Nagarajan, University of

California San Francisco, USA

\section{Reviewed by:}

Leslie J. Carver, University of

California San Diego, USA

Carme Junque, University of

Barcelona, Spain

\section{*Correspondence:}

Carole Scherling, School of

Psychology, University of Ottawa, 136

Jean Jacques Lussier, Vanier Hall,

\#2068, Ottawa, ON, Canada K1N 6N5.

e-mail:csche087@uottawa.ca

\begin{abstract}
Introduction: Cognitive deficits are a side-effect of chemotherapy, however pre-treatment research is limited. This study examines neurofunctional differences during working memory between breast cancer (BC) patients and controls, prior to chemotherapy. Methods: Early stage BC females (23), scanned after surgery but before chemotherapy, were individually matched to non-cancer controls. Participants underwent functional magnetic resonance imaging (fMRI) while performing a Visuospatial N-back task and data was analyzed by multiple group comparisons. fMRI task performance, neuropsychological tests, hospital records, and salivary biomarkers were also collected. Results: There were no significant group differences on neuropsychological tests, estrogen, or cortisol. Patients made significantly fewer commission errors but had less overall correct responses and were slower than controls during the task. Significant group differences were observed for the fMRI data, yet results depended on the type of analysis. BC patients presented with increased activations during working memory compared to controls in areas such as the inferior frontal gyrus, insula, thalamus, and midbrain. Individual group regressions revealed a reverse relationship between brain activity and commission errors. Conclusion: This is the first fMRI investigation to reveal neurophysiological differences during visuospatial working memory between $\mathrm{BC}$ patients pre-chemotherapy and controls. These results also increase the knowledge about the effects of $\mathrm{BC}$ and related factors on the working memory network. Significance: This highlights the need to better understand the pre-chemotherapy BC patient and the effects of associated confounding variables.
\end{abstract}

Keywords: pre-treatment effects, cognitive impairment, breast cancer, chemotherapy, functional magnetic resonance imaging, visuospatial working memory, surgery, stress

\section{INTRODUCTION}

Cognitive deficits, particularly in executive functioning domains such as working memory, are often reported in chemotherapytreated cancer populations. Such dysfunctions are increasingly described in adjuvant-chemotherapy breast cancer (BC) populations and are as such garnering more attention from the scientific community. Long-term survival is more frequently achieved with recent medical advances and is the primary focus in cancer populations. Meanwhile, secondary negative effects from treatment can potentially persist for years and considerably affect quality of life. To describe these self-reported cognitive deficits manifesting during and after chemotherapy, patients have coined the terms "chemofog" or "chemobrain." The reported incidence of chemotherapy-related impairments is highly variable with a range of $17-75 \%$ across studies (Correa and Ahles, 2007). Even with this inconsistency, these self-reports have been validated by neurospychological studies, which have largely confirmed that chemotherapy-treated patients perform more poorly on neurocognitive tests compared to non-exposed controls (Berglund et al., 1991; Wieneke and Dienst, 1995; Ahles et al., 1996, 2002; van Dam et al., 1998; Schagen et al., 1999; Brezden et al., 2000; Servaes et al., 2002; Gottschalk et al., 2003; Tchen et al., 2003; Castellon et al., 2004; Wefel et al., 2004; Mar Fan et al., 2005; Shilling et al., 2005; Bender et al., 2006; Hurria et al., 2006; Jenkins et al., 2006; Saykin et al., 2006; Scherwath et al., 2006; Wagner et al., 2006; Stewart et al., 2007). Additionally, neuroimaging studies have shown both structural and functional differences between patients and controls, even if the performance differences between the groups are very subtle (Brown et al., 1998; Saykin et al., 2003; Kreukels et al., 2005, 2006, 2008; Ferguson et al., 2007; Inagaki et al., 2007; Silverman et al., 2007; Abraham et al., 2008; Kesler et al., 2009; McDonald et al., 2010; de Ruiter et al., 2011; Deprez et al., 2011). In particular, these self-reported deficits, corroborated by neuropsychological and neuroimaging studies, are manifested mostly in domains such as processing speed and executive functioning, including working memory.

Working memory refers to the processes required to store and manipulate information for a short amount of time, subsequently followed by its retrieval (Baddeley and Hitch, 1974; Baddeley, 2000). This is an important component of executive functioning, requiring both the integrity of the prefrontal cortex, as well as its connections to the rest of the brain (Denkla, 1993; 
Fuster, 1997). The hippocampus is one of those associated areas required in several types of memory, including spatial memory (Glikmann-Johnston et al., 2008); visuospatial memory is particularly important due to its utility in day to day living. In addition to the frontal cortex and hippocampus, working memory is also subserved by the parahippocampal gyrus, the posterior parietal cortex, precuneus, and fusiform gyrus (Aguirre et al., 1996; Maguire et al., 1998, 2000; Ploner et al., 2000; Shipman and Astur, 2008).

Memory and attention have been target domains in neuroimaging studies of chemofog, with differences revealed between chemotherapy-treated patients (chemo+) and controls in frontal regions as well as associated areas. First, a delayed-recall word memory task during a [O-15] PET scan (Silverman et al., 2007) revealed that chemo + BC patients (minimum 5 years after treatment completion, self-reports of cognitive deficits) activated a larger portion of the inferior frontal cortex compared to other treatment BC patients (chemo-) and healthy participants. Interestingly, a [F-18] fluorodeoxyglucose-PET assessed lower resting brain metabolism in the left frontal gyrus and the contralateral cerebellum in chemo+ patients compared to controls. Additionally, lower resting metabolism in the frontal cortex correlated with the more impaired memory task performance. The authors suggest that the frontal cortex in chemo+ patients has to work harder to successfully perform the task.

Second, an functional magnetic resonance imaging (fMRI) study (Saykin et al., 2006) applied a working memory task in chemo+ BC patients, local therapy only BC patients and healthy participants. Working memory was assessed before and 1 month after treatment using an auditory $\mathrm{N}$-back task with variable processing load requirements $(0,1,2$, and 3 -back conditions). No group differences were observed at baseline, with the expected pattern of frontal, parietal, and cerebellar activations apparent during the most challenging load. Post-treatment fMRI assessments in chemo+ patients compared to both control groups uncovered increased activation in posterior frontal and parietal regions, as well as less bilateral activity in more anterior frontal regions.

Third, the largest and most recent fMRI study (de Ruiter et al., 2011) applied two executive functioning tasks in chemo+ (10 years post-treatment) and chemo- BC survivors. Chemo+ patients performed more poorly and had quicker reaction times while completing a Tower of London task compared to controls. Chemo+ patients additionally showed dorsal lateral prefrontal cortex (DLPFC) hyporesponsiveness and decreased activation in bilateral posterior parietal cortex. A paired-associates task was the second assessment and measured episodic memory. Chemo+ patients revealed hyporesponsiveness of the parahippocampal gyrus and the bilateral lateral posterior parietal cortex, left precuneus, right dorsal striatum, right inferior parietal cortex and left middle temporal gyrus compared to controls. This study suggests long-term effects of chemotherapy on executive functioning, linking DLPFC hypoactivation with impaired planning behavior often seen in chemo+ patients as well as increased impulsivity due to impaired attentional abilities.

Therefore, there are functional neural differences related to executive functioning between a chemotherapy-treated patient population and varied control groups, and one focused domain that seems to be affected is working memory. However, while both the term "chemofog" as well as the current research focus suggest a direct causal link between chemotherapy and cognitive deficits, other factors should be considered. An array of factors may contribute to variability between studies: nature of the control group, assessment tasks used, cross-sectional versus prospective studies, effects of anesthesia, and stress of cancer diagnosis. One study (Cimprich et al., 2010) has revealed pre-treatment group differences when conducting an fMRI study of verbal working memory in healthy controls and $\mathrm{BC}$ patients prior to chemotherapy. The authors revealed that patients had slower reaction times in the high-demand condition of the task as well as less accuracy in their responses. With increased cognitive demand, patients also showed larger activation in the right inferior frontal gyrus as well as additional components of attention/working memory circuitry in both hemispheres compared to controls. This study (Cimprich et al., 2010) highlights the importance of assessments of cancer patients before treatment as it suggests pre-treatment cognitive deficits. Without appropriate baseline assessments and scans, the effects of the disease itself or other baseline confounding variables could be mistaken for an effect of treatment. Similarly, these deficits could go undetected and lead to problems in day to day living for these women given the importance of executive functioning in such things as decision making and memory.

More attention to baseline differences between cancer patients and controls is warranted in order to tease out the contribution of other factors, either manifesting before chemotherapy commencement or exacerbating its effect. An important factor to consider is the presence of cancer itself, controlled by matching chemotherapy-treated patients (chemo+) to another cancer group receiving a different treatment (chemo-; Saykin et al., 2006; McDonald et al., 2010; de Ruiter et al., 2011). This practice is even more useful if the chemo- cancer group is applied as a second control group, along with healthy controls (Saykin et al., 2006; McDonald et al., 2010). However, the chemo+ and chemo- pairing presents differences in treatment. For example, the chemogroup may receive radiation treatment or anti-estrogen regimens, both which may contribute to fatigue (Noal et al., 2010) and be implicated in cognitive dysfunction in BC patients (Castellon et al., 2004). Regardless, the presence of cancer is an important factor to consider as ongoing studies have revealed elevated cytokine levels in cancer patients (Vardy et al., 2007) compared to healthy controls. These small proteins both interact with and modulate the activity of other cells in the body leading to inflammation, tissue destruction and/or disease. Of particular note is the problem of memory consolidation reported with high cytokine activity in the hippocampus (Maier and Watkins, 2003). The effects of cytokines can persevere for 6-60 months after diagnosis, with values being highest post-surgery (Vardy et al., 2007).

In addition to elevated cytokine levels post-surgery, cognitive decline linked to general anesthesia has been suggested. The latter is particularly potent in elderly patients $(60+)$ who are more prone to develop long-term cognitive deficits (Dodds and Allison, 1998; Dijkstra et al., 1999; Monk et al., 2008); exactly the age range of many cancer patients, especially in a $\mathrm{BC}$ population. Hence, cognitive deficits manifesting as side-effects of surgery that are not identified at baseline could be misattributed as chemofog deficits, especially in cross-sectional studies. One imaging 
study (McDonald et al., 2010) applied a covariate of "days between surgery date and baseline MRI scan" in a comparison between chemo + and chemo- groups. The covariate attenuated the statistical model but did not change the results, which led the authors to suggest that this speculated variable does not influence gray matter volume. However, this study compared two groups who had undergone surgery, both possibly exhibiting the same profile of elevated cytokine levels and deficits related to general anesthesia. More research is needed to understand post-surgery effects in cancer patients compared to healthy controls, and in a larger sample.

Another important factor to consider is the stress experienced by patients as a result of a cancer diagnosis and the follow up medical attention that then occurs. Stress, routinely measured in chemofog studies through self-reports, highly correlates with complaints of cognitive difficulties (van Dam et al., 1998; Schagen et al., 1999, 2002; Castellon et al., 2004; Shilling et al., 2005; Bender et al., 2006; Jenkins et al., 2006; Monk et al., 2008). With elevated stress, there is increased activity of the hypothalamic-pituitary-adrenal (HPA) axis which leads to increased glucocorticoid (GC) levels in the body (cortisol in humans). Increased GC levels (natural or a consequence of therapeutic GC regimens that are part of the cancer-related immunosuppressive therapy) have been shown to affect brain anatomy (Sapolsky, 1985; McEwen and Magarinos, 1997; Bremner et al., 1999) as well as memory functions, such as working memory (Lupien et al., 1999). In particular, regions in the brain such as the hippocampus are particularly affected by GC-mediated excitotoxicity as a consequence of its elevated number of GC receptors. In fact, smaller hippocampal volumes seen in high stress populations suggest potential negative effects of higher circulating GCs (Starkman et al., 1992; McEwen, 1999; Pruessner et al., 2005). One chemofog MRI study revealed transient smaller volumes in key cognitive regions such as the hippocampus in chemo $+\mathrm{BC}$ survivors compared to those treated with surgery alone. These structural differences were mirrored by lower scores in attention, visual memory, and concentration 1 year after chemotherapy (Inagaki et al., 2007). Additionally, GCs may have an indirect role in the development of cognitive deficits post-chemotherapy. Higher levels of circulating GCs during high stress, such as the occurrence of a cancer diagnosis and/or treatment, may contribute to increased capillary permeability of the blood brain barrier (Tuxen and Werner, 1994) which could lead to increased accessibility of chemotherapy agents to the brain. Therefore, increased GC levels could potentially exacerbate the adverse effects of chemotherapy on the brain.

Finally, another biological factor of importance in a typically older BC population is estrogen levels as chemotherapy-treatment is known to trigger early onset menopause (Goodwin et al., 1999; Lower et al., 1999) and many women are already post-menopausal at the time of diagnosis. Estrogen has long been considered to have a neuroprotective role in the brain, thereby helping maintain cognitive capacities. While the exact mechanism by which it exerts such protection remains unclear, there is likely an interaction between various neurotransmitter systems, neurotrophic factors as well as nuclear estrogen receptors. These are particularly present in areas such as the hippocampus and amygdala, both implicated in learning and memory (Shughrue and Merchenthaler, 2000; Behl,
2002; McEwen, 2002). After menopause, women report declining cognitive capacities and show decreased bilateral hippocampal volumes. This effect has been shown to be minimized by estrogen therapy (Lord et al., 2008).

The current study examined functional neural processing in newly diagnosed female BC patients (prior to chemotherapy commencement) compared to healthy controls completing a visuospatial working memory task. The visuospatial N-back task is a commonly used fMRI compatible cognitive assessment utilized to investigate visuospatial working memory and has been successfully applied in both healthy and clinical populations (Haberecht et al., 2001; Shackman et al., 2006; Smith et al., 2006, 2010; Haley et al., 2008; Alichniewicz et al., 2010). Brain regions that are particularly important for successful visuospatial working memory in healthy populations include the frontal cortex, hippocampus, parahippocampal gyrus, posterior parietal cortex, precuneus, fusiform gyrus, and cerebellum (Aguirre et al., 1996; Maguire et al., 1998, 2000; Ploner et al., 2000; Desmond et al., 2003; Shipman and Astur, 2008). A variety of speculated pre-chemotherapy confound variables were considered as covariates in the fMRI analysis, including: days since surgery, diurnal cortisol levels, estrogen measurements, depression and anxiety inventories, and task performance scores. It was hypothesized that addition of the above covariates may modify the analyses with no covariates (as seen in previous studies; McDonald et al., 2010), yet these may also uncover new baseline differences between patients and controls and/or expose heterogeneity within the patient sample itself. Such results would emphasize the multifactorial nature of the $\mathrm{BC}$ population and the need to include baseline comparisons to fully understand the repercussions of chemotherapy on cognitive processing.

\section{MATERIALS AND METHODS}

Twenty-three early stage BC patients were recruited through the Ottawa Hospital Regional Cancer Centre (average age: $51 \pm 8.5$ years, range: $35-64)$. Patients had undergone mastectomy or lumpectomy and were scheduled to commence a chemotherapy regimen. Inclusion criteria were: female, no previous cancer or cancer treatment, no unstable psychiatric/neurological illness, no history of substance abuse, scores of $<20$ on the Beck depression inventory (BDI-II; Beck et al., 1996) and $<15$ on the Beck anxiety inventory (BAI; Beck and Steer, 1990), fluency in English, and minimum grade 8 education. fMRI exclusion criteria included left-handedness, sight problems, claustrophobia, pacemaker, and metal implants. Healthy controls (average age: $49 \pm 9$ years, range: 30-62) were either nominated by a patient or recruited through posters/website ads and were each sex-, age-, and educationmatched to individual BC patients. Non-cancer controls also met the above inclusion and exclusion criteria and followed the same procedure as their index patient.

\section{NEUROPSYCHOLOGICAL ASSESSMENTS}

Each participant completed psychometric tests and MRI/fMRI scanning sessions. The 2.5 -h psychometric test battery included: social/medical history, self-report questionnaires of physical, emotional, and cognitive functioning, traditional pencil-and-paper neuropsychological tests, and a 30-min computerized cognitive test (CNSVS; https://www.cnsvs.com/). This computerized test, 
validated (Gualtieri and Johnson, 2006) in a broad age range and across clinical and non-clinical populations (including cancer patients), has also been proven sensitive in monitoring patient's cognitive status over time. In particular this battery measures: attention, reaction time, working memory, executive function, visual episodic memory, and verbal episodic memory. Raw neuropsychological data were converted to standardized scores, based on the means and SD of the healthy matched controls group. Summary scores were computed for several cognitive domains, as well as an overall cognitive summary calculated by averaging all the cognitive test scores. Cognitive domains were determined based on both rational and empirical grounds. Nineteen tests comprised in the neuropsychological battery from which the six following cognitive domains were extracted: 1. Verbal Memory [Hopkins Verbal Learning Test-Revised (HVLT-R; Brandt and Benedict, 2001) and CNSVS Verbal Memory Index], 2. Processing Speed [WAIS-III (Wechsler, 1997) Digit-symbol coding, WAIS-III Symbol Search, controlled oral word association; (Benton et al., 1994), CNSVS Processing speed index, CNSVS Executive Functioning index, CNSVS Reaction time index, and CNSVS Cognitive Flexibility index], 3. Attention [WAIS-III Digit Span, WAIS-III Letter-number sequencing, Paced Auditory Serial Attention Test (PASAT; Gronwall, 1997), CNSVS Sustained attention index, and Trail making Test, Part B (Army Individual Test Battery, 1944)], 4. Visual Memory [CNSVS Visual Memory index, Brief Visuospatial Memory Test-Revised (BVMT-R; Benedict, 1997), and CNSVS Working Memory index], 5. Reasoning (CNSVS Reasoning index), and 6. Verbal Short-term Memory (Auditory Consonant Trigrams; Boone et al., 1990). At the baseline scanning session, each participant also completed the Rosenberg self-esteem questionnaire (RSE; Rosenberg, 1985) and the Questionnaire for Competence and Control (FKK; Krampen, 1991).

Demographic, clinical neuropsychological and self-esteem data were analyzed by Two-tailed independent $t$-tests using Statistical Package for the Social Sciences version 18.0 program (SPSS v. 18.0, Chicago, IL, USA). A correlational analysis was also conducted to reveal any relationships between factors.

\section{ASSESSMENT OF BIOLOGICAL MARKERS}

Baseline blood work [hemoglobin, white blood cell (WBC), and neutrophil counts] were obtained from patient's hospital records. Diurnal free cortisol levels were assessed via salivary sampling (Kirschbaum and Hellhammer, 1994). Each at-home sampling kit used for this study contained 14 cotton salivettes for cortisol sampling and detailed instructions. Participants were instructed to follow a rigid sampling schedule for two consecutive and typical weekdays. Cortisol was sampled at seven time points for each of the 2-days at: Awakening, $30 \mathrm{~min}$ after awakening, $1 \mathrm{~h}$ after awakening (after this sample, participants may consume food and drink), 10a.m., 2p.m., 6p.m., and 9p.m. Such a sampling schedule provides a circadian cortisol profile for each participant (patients and controls), an important factor to consider since cortisol varies according to circadian rhythms and such cycles are seen to be affected in high stress and clinical populations (Pruessner et al., 1997, 1999, 2003; MacHale et al., 1998; Backhaus et al., 2004; Monteleone and Maj, 2009). Participants were instructed to keep the completed samples in a freezer until the next appointment. The saliva samples were analyzed for cortisol using ELISA techniques (as described by Salimetrics at salimetrics.com). Intra- and interassay variabilities (CVs) for datapoints (excluding outliers) were less than 10 and 15\%, respectively. The area under the curve from ground (AUCg) calculation was computed in accordance to the formulas described by Pruessner et al. (2003) in order to analyze salivary cortisol differences. AUCg was computed and two-tailed independent $t$-tests were applied using SPSS v. 18.0.

Estrogen samples were also collected as part of the home sampling kits by collecting pure spit. This was scheduled to be completed at the 10a.m. mark for each of the two consecutive days. The saliva samples were analyzed for estradiol using ELISA techniques (described by Salimetrics at salimetrics.com). Intra-assay coefficients of variation on one estrogen measurement/participant were less than $0.43(\mathrm{CV}(\%)=4.03$, Max: 19, and Min 0.148). Group differences in estrogen levels were assessed by a two-tailed independent $t$-test using SPSS v. 18.0.

\section{SCANNING SESSIONS}

Brain images were acquired using a 1.5-T Siemens Magnetom Symphony MR scanner. Subjects were instructed to lie on their back with their head secured in a standard head holder and to stay as still as possible when in the scanner. A gradient echo localizer was acquired and used to prescribe a subsequent 3D FLASH [Fast Low Angle Shot, a spoiled gradient sequence; TR/TE 22/9.2 ms, flip angle $30^{\circ}$, field of view (FOV) $256 \times 256 \mathrm{~mm}$ scan]. Whole brain echo planar fMRI based on the blood oxygen level dependent (BOLD) effect was performed using a gradient echo pulse sequence (TR/TE 3000/40 ms, flip angle $90^{\circ}$, FOV $250 \mathrm{~mm} \times 187.5 \mathrm{~mm}$ $64 \times 64$ matrix, slice thickness $5 \mathrm{~mm}, 27$ axial slices, bandwidth $2430 \mathrm{~Hz}$ per pixel).

\section{fMRI VISUOSPATIAL N-BACK TASK}

The task was projected through an LCD projector from a computer in the control room, and presented on a rear projection screen placed near the end of the patient table. A mirrored head coil allowed the participant to view the task while lying in the scanner. In order to increase visibility, all lighting in the scanning room was off. Participant responses, in the form of button presses on a response pad, were recorded via a MRI compatible fiber optic device (Lumina LP-400, Cedrus).

The Visuospatial N-back was a block-design task used to measure visuospatial working memory processes while in the scanner. Stimuli were presented as white circular $(\mathrm{O})$ targets in nine different positions on a solid black background. Participants were instructed to use the response pad with their right hand, and to press the correct key as quickly and accurately as possible, using the right index finger. They were encouraged not to dwell on previous mistakes. The scanning session began with an initial rest epoch of $9 \mathrm{~s}$ in order to allow longitudinal magnetic relaxation (T1 effects) to stabilize. Images collected during this initial rest epoch were excluded from the image analyses.

The Visuospatial N-back procedure involved two conditions of target presentations, with the circular stimuli being presented one at a time on the screen for $240 \mathrm{~ms}$ (interstimulus interval of $1760 \mathrm{~ms}$ ). The first condition, "Press for center O," required the participant to press a button when an $\mathrm{O}$ appeared in the center 
of the screen and to refrain from pressing when it appeared at another location on the screen. The second condition, "Press for 2back," required the participant to press when the target appeared at the same location on the screen as the stimuli presented two trials prior and to refrain from pressing for all other presentations. Both of these conditions were presented in 12 epochs of 33 s. Each epoch began with a 3-s instruction screen, followed by the presentation of 15 stimuli. There were two $24 \mathrm{~s}$ rest epochs, one in the middle of the task and another at the very end. During instruction epochs, the participant was to either "Press for center O" and "Press for 2back." During rest epochs, the word "REST" was presented on the screen, instructing the participant that no motor response was required. Once in the scanner, six blocks of each condition, "Press for center O" and "Press for 2back" epochs, were presented in a counterbalanced order, starting with the "Press for center O" condition. This task lasted 453 s. After the completed task, all participants were asked to rate the difficulty of the task on a 5-point Likert Scale to investigate metacognitive capacity. (On a scale of zero to five how difficult did you find this task. Zero means that no mental effort was required and five means that it was painfully difficult.)

\section{fMRI PERFORMANCE PARAMETERS AND ANALYSES}

Errors of commission included any incorrect response following a stimulus (e.g.,: pressing a target not presented in the center in the "Press for center O" condition or pressing for a target that was not presented two prior while performing the "Press for 2back" condition) within $900 \mathrm{~ms}$ of stimulus presentation. Omission errors were defined as a failure to respond to a target stimulus within the same time frame. All accurate responses occurring within $900 \mathrm{~ms}$ of stimulus presentation were used to calculate the percent correct scores and the mean reaction times for both conditions.

Reaction times for each response in the scanner, metacognition scores, and both errors of commission and omission (or percent correct scores) were analyzed. Group differences were computed by two-tailed independent sample $t$-tests using SPSS v. 18.0. A correlational analysis was also applied to reveal if there was a relationship between the cognitive impression of task difficulty and actual task performance.

\section{IMAGE PROCESSING}

Statistical Parametric Mapping 8 (SPM8) was used to post-process the fMRI data and to perform the statistical analyses specific to this dataset. The functional images were reconstructed and whole brain images were realigned to correct for motion by employing the procedure of Friston et al. (1995). Then, images were spatially normalized to match the echo planar imaging (EPI) template provided in SPM8. Following spatial normalization, images were smoothed with a $10-\mathrm{mm}$ full-width at half-maximum Gaussian filter.

First level analyses were performed for each participant using these images representing the two task conditions and the rests. Motion correction was applied as a regressor for all first level analyses. Visuospatial working memory processes were isolated by subtracting the averaged "Press for center O" images from the averaged "Press for 2back" images (condition labeled as "2back-O"), creating one image per participant. Two additional contrasts were also computed at the first level: "press for center O - rest" (or "O-rest") and "press for 2 back - rest" (or "2back-rest"). These processed within-subject images were then entered into the second level analyses for group comparisons between BC patients and controls. Second level analyses included three different assessments. First, a two-way independent group $t$-test of the " 2 back-O" contrast was executed without covariates, and then separate $t$-tests were performed with suspected confound variables applied as covariates in order to extract their possible individual effects. Second, flexible factorial assessments of both "O-rest" and "2back-rest" contrasts were conducted in order to assess if the rest condition itself could drive group differences. Third, regression analyses along days since surgery in the patient group and fMRI task errors of commission, percent correct, and reaction times in both controls and patients were performed for the "2back-O" contrast to assess if there was group heterogeneity in visuospatial working memory caused by these factors.

Whole brain and ROI investigations for both $t$-test and regression analyses were conducted at a set threshold of $p_{\text {uncorr }}=0.001$, with a cluster-wise correction at $p_{\mathrm{FWE}}=0.05$. Whole brain fMRI, more stringent than ROI analyses, measures activation differences over the entire brain, thus any effect size apparent in small areas will be diluted. ROIs investigated in this study were extracted from several sources including, widespread brain areas reported as affected in previous post-chemotherapy structural and functional studies (Kreukels et al., 2005, 2006, 2008; Saykin et al., 2006; Inagaki et al., 2007; Silverman et al., 2007; Abraham et al., 2008; McDonald et al., 2010; Raffa and Tallarida, 2010; Deprez et al., 2011), regions related to the covariates used and areas with a role in working memory (Smith et al., 2010). Bilateral ROIs considered included: frontal lobe, precentral gyrus, cingulate gyrus, parahippocampus, hippocampus, insula, thalamus, basal ganglia, temporal lobe, parietal lobe, precuneus, occipital lobe, brainstem, and cerebellum. Significant results presented are both un-adjusted and adjusted for multiple comparisons. A Bonferroni adjustment with 28 comparisons lowers the alpha to $3.57^{-5}$ with a significant $Z$ value now of 3.97. Meanwhile, whole brain investigations for flexible factorial analyses were conducted at a set threshold of $p=0.05$ corrected, with a cluster-wise correction at $p_{\mathrm{FWE}}=0.05$.

\section{RESULTS}

\section{DEMOGRAPHIC AND CLINICAL DATA}

Group comparisons and correlations were computed to investigate any relationships between the demographic and neuropsychological factors. Table 1 summarizes demographic and clinical characteristics for all participants and Table 2 shows the significant correlational values. Estimated IQ was assessed by the Wechsler Adult Reading Task, and no significant group differences between patients and controls were revealed $[t(44)=-1.312, p=0.197]$. No significant differences existed between groups concerning menopausal status, with half the sample being post-menopausal. Finally, there were no significant group differences in any of the six cognitive domains or on self-esteem measures. The BDI revealed a larger mean score in patients (8.3) compared to controls (3.8) $[t(44)=2.299, p=0.026]$. Similarly, the BAI showed larger scores in patients (7.8) compared to controls $(4.1)[t(44)=2.090$, 
Table 1 | Demographic and clinical data.

\begin{tabular}{|c|c|c|}
\hline Factors & Patients ( $n=23$ ) & Controls $(n=23)$ \\
\hline Age & $51.5(8.47)$ & $50.4(8.82)$ \\
\hline Estimated IQ & $105.8(9.05)$ & $109.22(8.43)$ \\
\hline \multicolumn{3}{|l|}{ Education Level } \\
\hline High school & 2 & 3 \\
\hline College & 10 & 11 \\
\hline Bachelors & 6 & 3 \\
\hline Masters & 3 & 6 \\
\hline $\mathrm{PhD}$ & 1 & 0 \\
\hline \multicolumn{3}{|l|}{ Marital status } \\
\hline Married/common law & 19 & 14 \\
\hline Single & 0 & 3 \\
\hline Separated/divorced & 2 & 6 \\
\hline Widowed & 2 & 0 \\
\hline Days since surgery & $47.6(\max 71, \min 28)$ & - \\
\hline \multicolumn{3}{|l|}{ Cancer stage } \\
\hline 1 & 4 & - \\
\hline $2 a$ & 10 & - \\
\hline $2 b$ & 5 & - \\
\hline За & 4 & - \\
\hline \multicolumn{3}{|l|}{ Menopausal status } \\
\hline Menstruating & 8 & 9 \\
\hline Perimenopausal & 3 & 2 \\
\hline Post-menopausal & 12 & 12 \\
\hline$\left.\mathrm{BDI}\right|^{*}$ & $8.3(8.92)$ & $3.78(3.06)$ \\
\hline $\mathrm{BAI}{ }^{*}$ & $7.81(7.25)$ & $4.13(4.35)$ \\
\hline \multicolumn{3}{|c|}{ Neuropsychological domain scores } \\
\hline Verbal memory & $-0.24(1.19)$ & - \\
\hline Processing speed & $-0.11(0.91)$ & - \\
\hline Attention & $-0.26(0.86)$ & - \\
\hline Visual memory & $0.22(0.78)$ & - \\
\hline Reasoning & $-0.35(1.48)$ & - \\
\hline Verbal short-term memory & $-0.17(0.65)$ & - \\
\hline
\end{tabular}

* Significant group difference (two-way independent $t$-test, $p<0.05$ ). Age, estimated IQ, BDI, BAl, domain scores= mean (SD); educational level, marital status, menopausal status $=n$; days since surgery: number of days between surgery and baseline scan; domain scores = standardized to controls.

1Q, intelligence quotient; BDI, Beck depression inventory; BAI, Beck anxiety inventory. $p=0.042]$. As expected, BDI scores positively correlated with BAI scores $[r(46)=0.342, p=0.020]$ in all participants.

\section{BIOLOGICAL MEASURES}

Patient blood work revealed average values in the normal range: hemoglobin (HGB): $134 \mathrm{~g} / \mathrm{L}$, WBC: $7 \times 10^{9} / \mathrm{L}$, Neutrophils: $4.21 \times 10^{9} / \mathrm{L}$. Independent $t$-tests between patients and controls did not reveal significant differences on salivary estrogen levels $[t(25)=0.835, p=0.412]$ with average values (SD) being 6.07 (3.89) and 4.98 (2.89), respectively. Each subject's diurnal cortisol index ranged from 0 to $0.973 \mu \mathrm{g} / \mathrm{dL}$, mean $(\mathrm{SD})=0.144$ (0.159). AUCg values of diurnal salivary cortisol $[t(25)=-1.308$, $p=0.203]$ were not significantly different between groups, with average AUCg (SD) at 12.7 (5.41) and 16.1 (8.68), respectively for patients and controls. See Figure 1 for graph of diurnal cortisol rhythms for BC patients and matched controls.

\section{fMRI TASK PERFORMANCE DATA}

Group differences were revealed concerning reaction times for the "Press for 2back" condition $[t(44)=2.520, p<0.015]$ with patients showing slower response times than controls [mean (SD): $547.77 \mathrm{~ms}$ (78.98) and $486.25 \mathrm{~ms}$ (86.40)]. Also, errors of commission scores significantly differentiated patients and controls $[t(44)=-2.320, p<0.025]$ with patients making less erroneous presses than controls [mean (SD; max, min): $0.39(0.66 ; 2,0)$ and $1.18(1.49 ; 5,0)]$. Meanwhile, patients committed more errors of omission than controls and performed with 73 and $82 \%$ accuracy, respectively. However, this was not a significant difference $[t(44)=-1.779, p>0.082]$. No other group differences were revealed. Overall, metacognition scores across groups correlated with errors of commission $[r(46)=0.467, p=0.001]$, percentage of correct responses [ $r(46)=-0.362, p=0.013]$, BDI $[r(46)=0.632, p=0.000]$, and BAI $[r(46)=1, p=0.000]$.

\section{fMRI TASK IMAGE ANALYSIS Two-way independent group t-test (2back-0)}

Independent $t$-test results of the 2 back-O contrast (Whole brain $p$ values, MNI coordinates, ROI $p$ values, region, cluster extent, $T$ and $Z$ values) are presented in Table 3 , at threshold $p_{\text {uncorr }}<0.001$ with $p_{\mathrm{FWE}}=0.05$ cluster-wise correction. The data was first analyzed without covariates. To control for any effects of "contributing" factors, the following were individually added as covariates in

Table 2 | Demographic and neuropsychological data correlation for all study participants.

\begin{tabular}{llllll}
\hline $\begin{array}{l}\text { Correlated } \\
\text { factors }\end{array}$ & Processing speed & Attention & Visual memory & Reasoning & $\begin{array}{l}\text { Verbal short-term } \\
\text { memory }\end{array}$ \\
\hline Age & $r(46)=-0.531, p<0.001$ & $r(46)=-0.311, p=0.035$ & & $r(46)=-0.418, p=0.004$ & \\
IQ & $r(46)=0.447, p=0.002$ & $r(46)=0.486, p=0.001$ & $r(46)=0.332, p=0.026$ & $r(46)=0.295, p=0.049$ & $r(46)=0.459, p=0.002$ \\
Verbal memory & & & $r(46)=0.353, p=0.016$ & \\
Attention & $r(46)=0.551, p=0.000$ & & & & \\
Visual memory & $r(46)=0.314, p=0.033$ & & & $r(46)=0.596, p=0.000$ \\
BDI & $r(46)=-0.368, p=0.012$ & & & $r(46)=-0.362, p=0.013$ \\
BAI & & &
\end{tabular}

$r=$ Pearson correlations. No other significant correlations were revealed. 


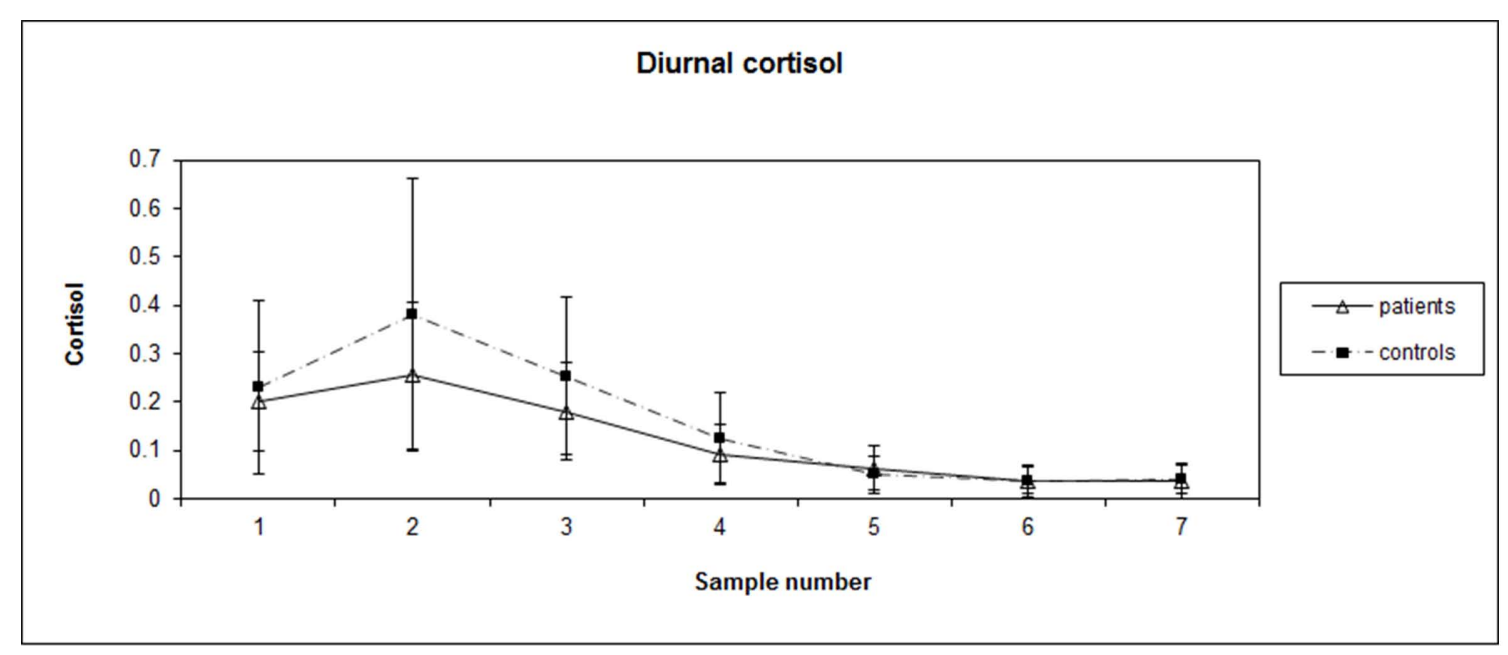

FIGURE 1 | Diurnal cortisol rhythms for breast cancer patients and matched controls.

Table 3 | Significant $\boldsymbol{t}$-test results comparing patients and controls for the 2 back-0 contrast.

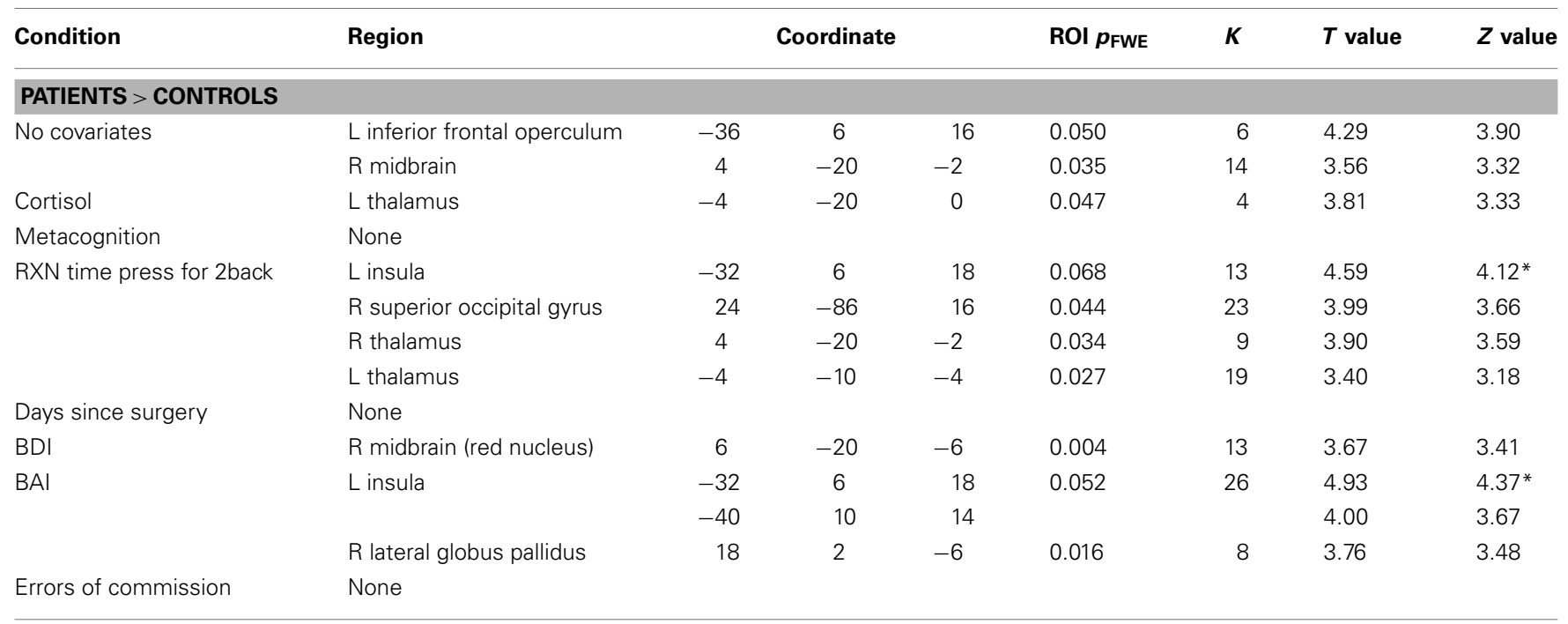

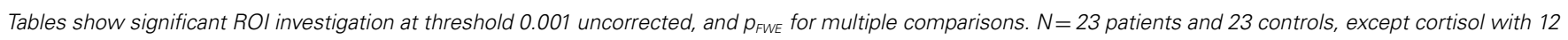
patients and 15 controls. $R$, right; $L$, left; $K$, cluster size; *indicates significance at Bonferroni adjustment for multiple comparisons.

the analyses: cortisol, metacognition, "Press for 2back" condition reaction time, days since surgery, BDI scores, BAI scores, and the number of errors of commission (See Figure 2).

Fixed-effects in patients and controls concerning visuospatial working memory are presented in the Table A1 in Appendix. When comparing groups, patients showed increased activity compared to controls in the left inferior frontal operculum and right midbrain. The addition of days since surgery as a covariate eliminated any group differences. Group differences when considering reaction time as a covariate showed increased activation in patients compared to controls in the right super occipital gyrus and bilateral thalamus. Patients revealed increased activity compared to controls in the left insula as well as the right lateral globus pallidus with the addition of BAI scores and in the red nucleus with the addition of BDI scores. When cortisol was added as a covariate, patients showed increased activity in the left thalamus. Finally when metacognitive scores of performance during the task were considered, patients revealed larger activity in the right thalamus compared to controls. Overall, patients consistently revealed larger activity compared to controls, and these regions changed according to the covariate in question.

\section{Flexible factorial (0-rest and 2back-rest)}

All flexible factorial results for the O-rest and the 2back-rest contrasts (Whole brain $p$ values, MNI coordinates, ROI $p$ values, region, $T$ and $Z$ values) are presented in Table 4 , at threshold corrected $p_{\text {FWE-corrected }}<0.05$ with $p_{\text {FWE }}=0.05$ set for multiple comparisons. See Figure 3. 


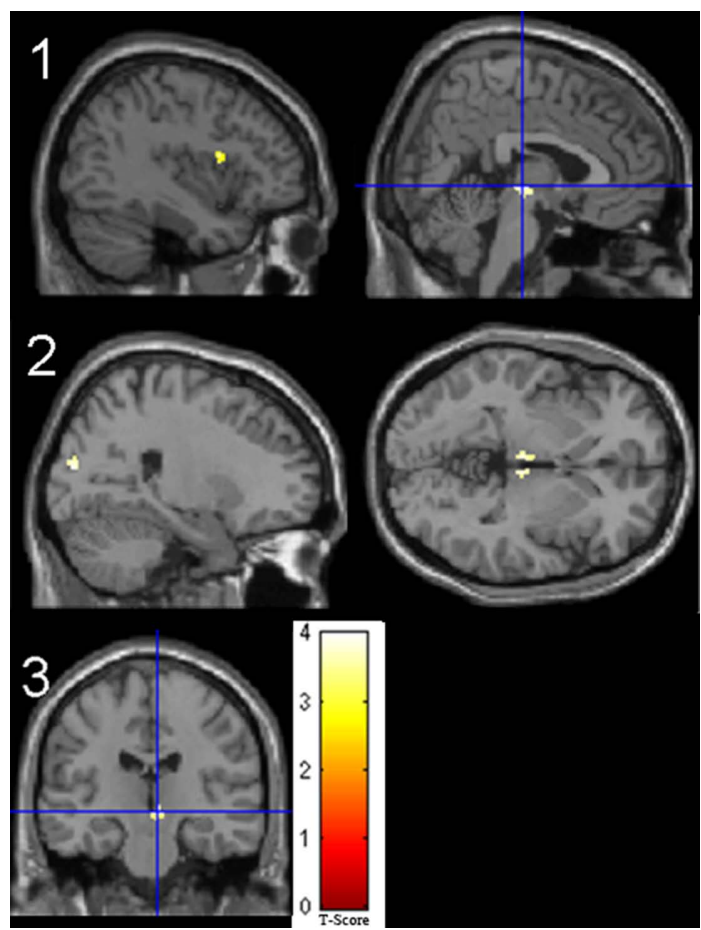

FIGURE 2 | Significant $\boldsymbol{t}$-test results for patients > controls for the 2back-0 contrast. 1. No covariate (left frontal operculum, right midbrain); 2. Reaction time as covariate (bilateral thalamus, right superior occipital gyrus); 3. BDI as covariate (right red nucleus).

There was an overlap in the regions where patients demonstrated more neural activity compared to controls in both the 0 -rest and the 2back-rest conditions. However, the 2back-rest condition additionally showed increased activity in the left hippocampus, right supramarginal gyrus, right angular gyrus, right thalamus, right lingual gyrus, and right precuneus in patients compared to controls.

There was also overlap in brain regions where patients showed significantly less activation compared to controls for both the 0 rest and the 2back-rest conditions. However, there were additional, non-overlapping regions for each condition as follows. For the 0 -rest condition, there was increased activity in the right precentral, left lentiform nucleus, right putamen, left middle occipital gyrus, right cuneus, and left cerebellar tonsil. For the 2back-rest condition, there were additional differences in the right insula, left middle temporal gyrus, left superior temporal gyrus, and left superior parietal lobule.

\section{Regression (2back-0)}

Regression results of the 2 back-O contrast along days since surgery as well as fMRI task errors of commission, reaction times, and percent correct (Whole brain $p$ values, MNI coordinates, ROI $p$ values, region, cluster extent, $T$ and $Z$ values) are presented in Table 5, at threshold $p_{\text {uncorr }}<0.001$ with $p_{\text {FWE }}=0.05$ clusterwise correction. Within-group variability in visuospatial working memory was revealed in both groups via regressions along errors of commission with widespread activations. However, while patients mostly revealed positive correlations between brain activity and these errors (more activity was associated with more errors), controls revealed negative correlations (more activity was associated with less errors). Regressions along percentage of correct responses was only significant in the control group, with visuospatial working memory activity increasing in the left inferior frontal gyrus, superior, and medial orbitofrontal gyrus as well as parahippocampus with increased performance.

\section{DISCUSSION}

Post-chemotherapy cognitive impairments reported by cancer patients are widely acknowledged and these can seriously affect quality of life. While post-chemotherapy investigations are becoming more common, only a few studies have investigated and reported on existing pre-treatment cognitive differences (Gualtieri and Johnson, 2006; Haley et al., 2008). The purpose of the current study was to further pre-chemotherapy baseline investigations through the examination of neural processing during visuospatial working memory in BC patients and well-matched healthy controls. This was the first study to investigate visuospatial working memory abilities in BC patients using an N-back task in an fMRI setting. Also, this study is novel in its approach to control for multiple variables that may contribute to group differences in cognitive processing prior to chemotherapy.

Working memory deficits are apparent after chemotherapy (Denkla, 1993; Correa and Ahles, 2007), however, the results from this visuospatial working memory study suggest that these deficits are manifesting at baseline. Carry-over of these effects to post-treatment assessments could lead to them being mistakenly attributed as a side-effect of chemotherapy. It is also apparent from these study results that the BC population is a multifaceted group requiring consideration of many potentially confounding variables. It is possible that the combination of these variables with chemotherapy-treatment could exacerbate already existing deficits. Therefore, before post-chemotherapy deficits are assessed in BC patients, it is essential to better understand these pre-treatment patients.

This study demonstrates that BC patients, prior to chemotherapy, have different patterns of neural activity than controls when completing a visuospatial working memory task. The particular results vary depending on the statistical protocol implemented and the covariates included, highlighting the need for rigorous analyses procedures. The rigorous analyses conducted in this study collectively enabled a systematic investigation of the many variables playing a role at baseline. Such varied statistical investigations have not been applied in previous studies of BC patients.

An ROI analysis $t$-test, focused on regions reported by postchemotherapy studies, uncovered that BC patients show more neural activity related to visuospatial working memory in the left inferior operculum and right midbrain compared to controls. Activation of the inferior frontal gyrus has been reported in the visuospatial N-back task for the 2back-0back analysis (Jonides et al., 1993; Smith et al., 1996; Carlson et al., 1998) as well as the insula (at the frontal operculum; Carlson et al., 1998). The left inferior frontal gyrus shows greater activation in working memory tasks with long delays compared to short delays, thereby indicating that this region activates more with increased challenges 
Table 4 | Significant flexible factorial results comparing patients and controls for the 2 back-rest and 0-rest contrasts.

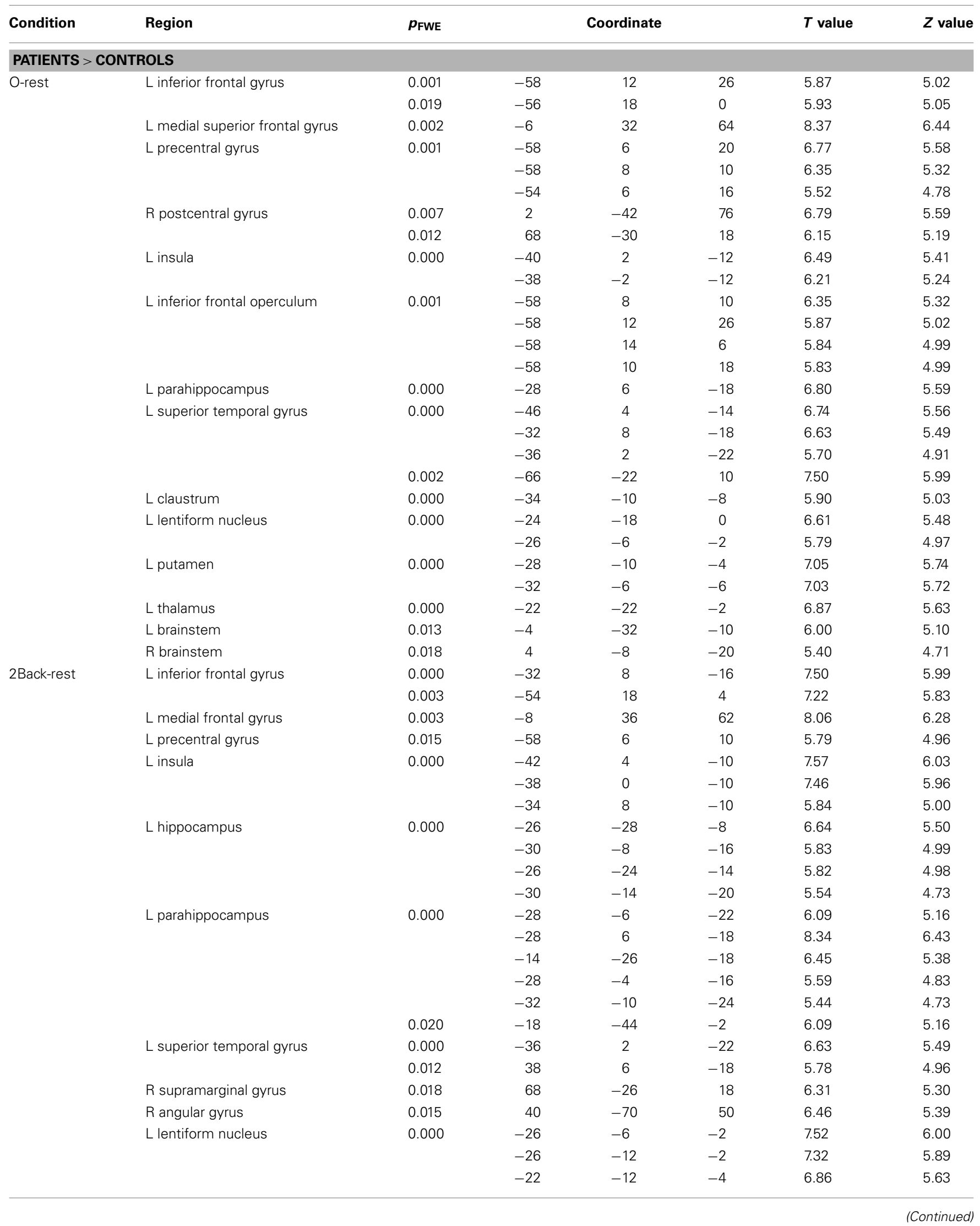


Table 4 | Continued

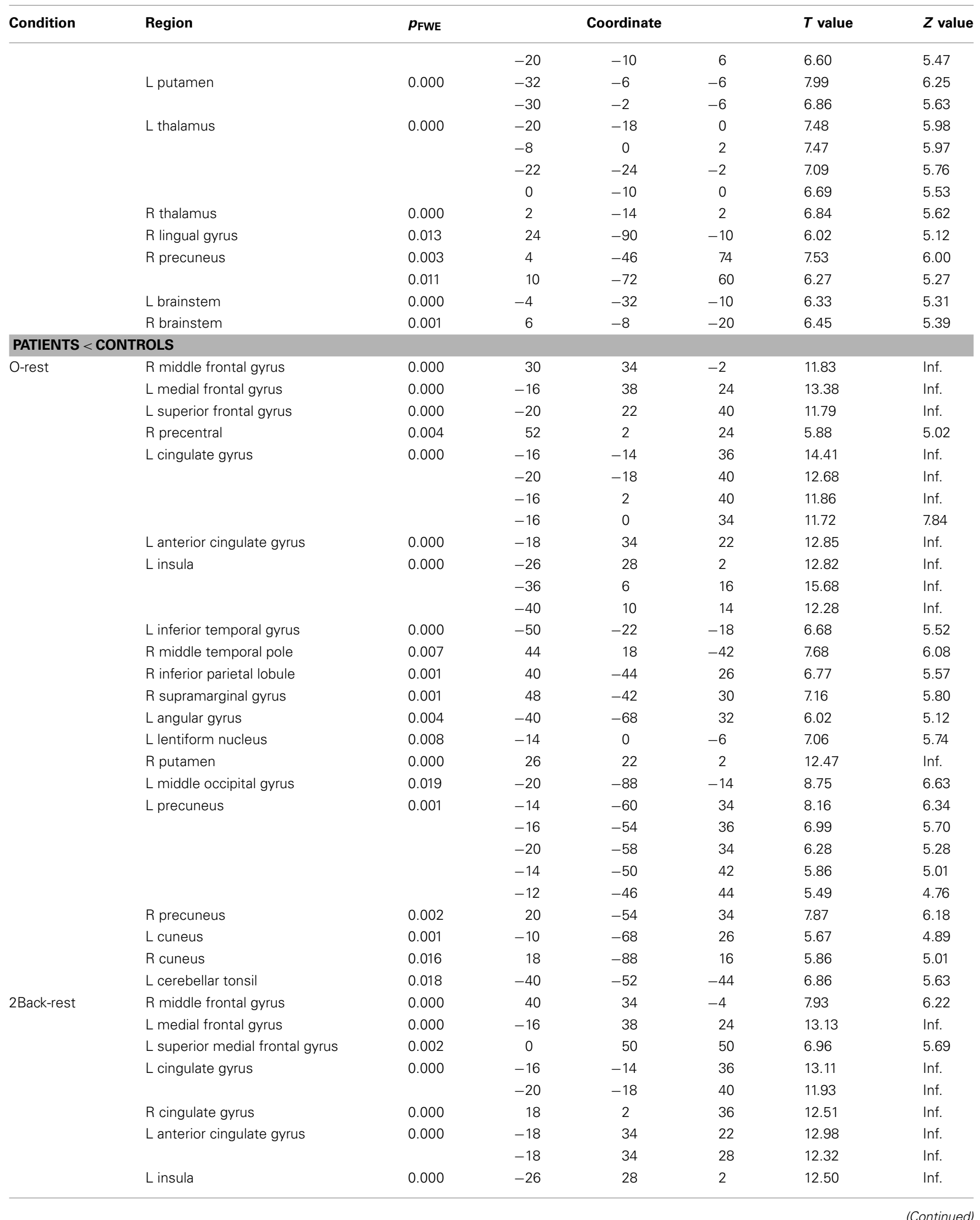


Table 4 | Continued

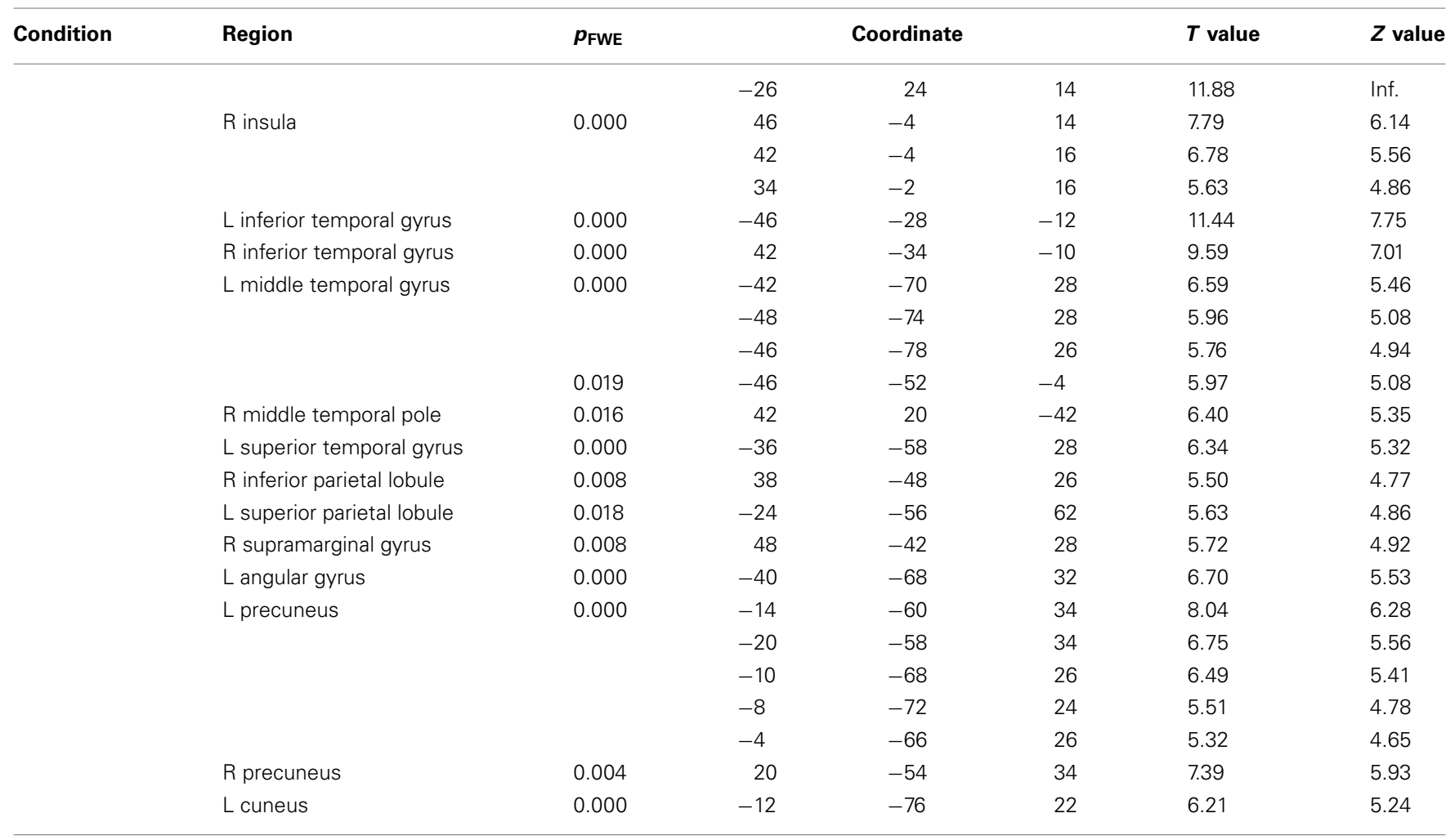

Tables show significant activations at $p_{F W E}=0.05 . \mathrm{N}=23$ patients and 23 controls, except cortisol with 12 patients and 15 controls. $R$, right; $L$, left.
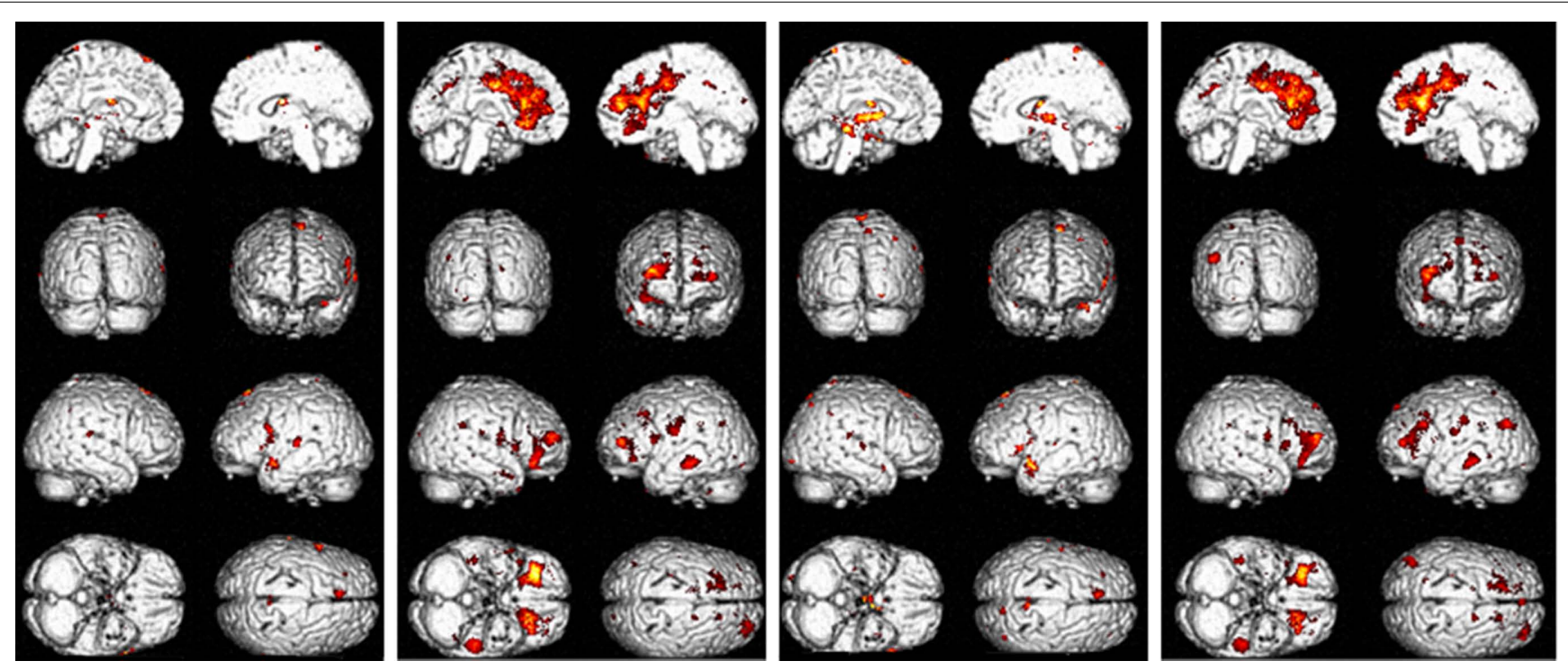

FIGURE 3 | Significant flexible factorial results for 0-rest and 2back-rest contrasts. (1) Patients > controls 0-rest contrast; (2) patients < controls 0-rest contrast; (3) patients > controls 2back-rest contrast; (4) patients < controls 2back-rest contrast.

to working memory processing (Barch et al., 1997). Additionally the pars opercularis of the inferior frontal gyrus is active when people suppress motor responses (Aron et al., 2007), and stimulation causes changes in motor cortical activity (Buch et al., 2010).
Overall, increased activation in the frontal operculum is interesting as patients reveal slower reaction times compared to controls. This may indicate that patients find the task more challenging than controls (as suggested by the percent correct scores), leading 
Table 5 | Significant regression results for errors of commission, reaction times, and days since surgery.

\begin{tabular}{|c|c|c|c|c|c|c|c|c|}
\hline \multirow{2}{*}{$\begin{array}{l}\text { Condition } \\
\text { PATIENTS }\end{array}$} & \multirow[t]{2}{*}{ Region } & \multirow[t]{2}{*}{$\mathrm{ROI} p_{\mathrm{FWE}}$} & \multirow[t]{2}{*}{$\kappa$} & \multicolumn{3}{|c|}{ Coordinate } & \multirow[t]{2}{*}{$T$ value } & \multirow[t]{2}{*}{$Z$ value } \\
\hline & & & & & & & & \\
\hline \multirow[t]{37}{*}{ Errors of commission+ } & L supplemental motor & 0.038 & 34 & -12 & -4 & 74 & 4.63 & 3.80 \\
\hline & & & & -2 & -8 & 76 & 4.05 & 3.44 \\
\hline & L precentral & 0.038 & 82 & -58 & -2 & -12 & 5.40 & $4.23 *$ \\
\hline & $\mathrm{L}$ anterior cingulate & 0.053 & 14 & -8 & 24 & 24 & 4.70 & 3.84 \\
\hline & $\mathrm{R}$ posterior cingulate & 0.017 & 50 & 12 & -58 & 4 & 4.83 & 3.92 \\
\hline & $\mathrm{L}$ insula & 0.030 & 55 & -26 & 24 & -4 & 5.81 & $4.44^{*}$ \\
\hline & & & & -26 & 20 & 6 & 5.27 & $4.16^{*}$ \\
\hline & L hippocampus & 0.008 & 108 & -32 & -20 & -14 & 4.51 & 3.73 \\
\hline & & & & 34 & -24 & -12 & 3.98 & 3.39 \\
\hline & L parahippocampus & 0.041 & 41 & -34 & -50 & -6 & 4.18 & 3.53 \\
\hline & $\mathrm{R}$ parahippocampus & 0.010 & 100 & 16 & -26 & -10 & 5.31 & $4.18^{*}$ \\
\hline & & & & 16 & -26 & -14 & 5.20 & $4.12^{*}$ \\
\hline & & & & 20 & -30 & -4 & 4.86 & 3.94 \\
\hline & & & & 26 & -36 & -4 & 4.40 & 3.66 \\
\hline & & & & 22 & -36 & -6 & 4.24 & 3.56 \\
\hline & & & & 22 & -40 & -4 & 4.16 & 3.14 \\
\hline & & & & 32 & -38 & -6 & 3.59 & 3.14 \\
\hline & L inferior temporal & 0.024 & 94 & -40 & -36 & -14 & 4.37 & 3.64 \\
\hline & & & & 34 & -72 & -14 & 4.68 & 3.83 \\
\hline & & & & 30 & -72 & -16 & 4.58 & 3.77 \\
\hline & L supramarginal & 0.019 & 56 & -54 & -38 & 26 & 5.35 & $4.20 *$ \\
\hline & $L$ thalamus & 0.001 & 318 & -12 & -22 & 6 & 5.91 & $4.49 *$ \\
\hline & & & & -2 & -14 & 14 & 5.31 & $4.18^{*}$ \\
\hline & & & & -6 & -8 & 2 & 4.09 & 3.47 \\
\hline & $\mathrm{R}$ thalamus & 0.000 & 386 & 14 & -16 & 2 & 4.99 & $4.01 *$ \\
\hline & & & & 18 & -24 & -2 & 4.67 & 3.82 \\
\hline & & & & 2 & -14 & 12 & 4.62 & 3.79 \\
\hline & L inferior occipital & 0.021 & 41 & -36 & -74 & -12 & 4.18 & 3.53 \\
\hline & $\mathrm{R}$ middle occipital & 0.047 & 41 & 52 & -62 & -10 & 4.13 & 3.50 \\
\hline & L lingual & 0.013 & 118 & -12 & -54 & -4 & 4.24 & 3.57 \\
\hline & & & & -28 & -56 & -6 & 4.17 & 3.52 \\
\hline & & & & -20 & -56 & -4 & 3.79 & 3.27 \\
\hline & $\mathrm{R}$ lingual & 0.001 & 368 & 12 & -62 & 2 & 4.89 & 3.95 \\
\hline & & & & 20 & -44 & -2 & 4.49 & 3.72 \\
\hline & & & & 12 & -54 & -4 & 3.97 & 3.39 \\
\hline & & 0.028 & 67 & 8 & -86 & -12 & 4.27 & 3.59 \\
\hline & $\mathrm{L}$ brainstem & 0.006 & 190 & 0 & -30 & -18 & 4.29 & 3.60 \\
\hline
\end{tabular}


Table 5 | Continued

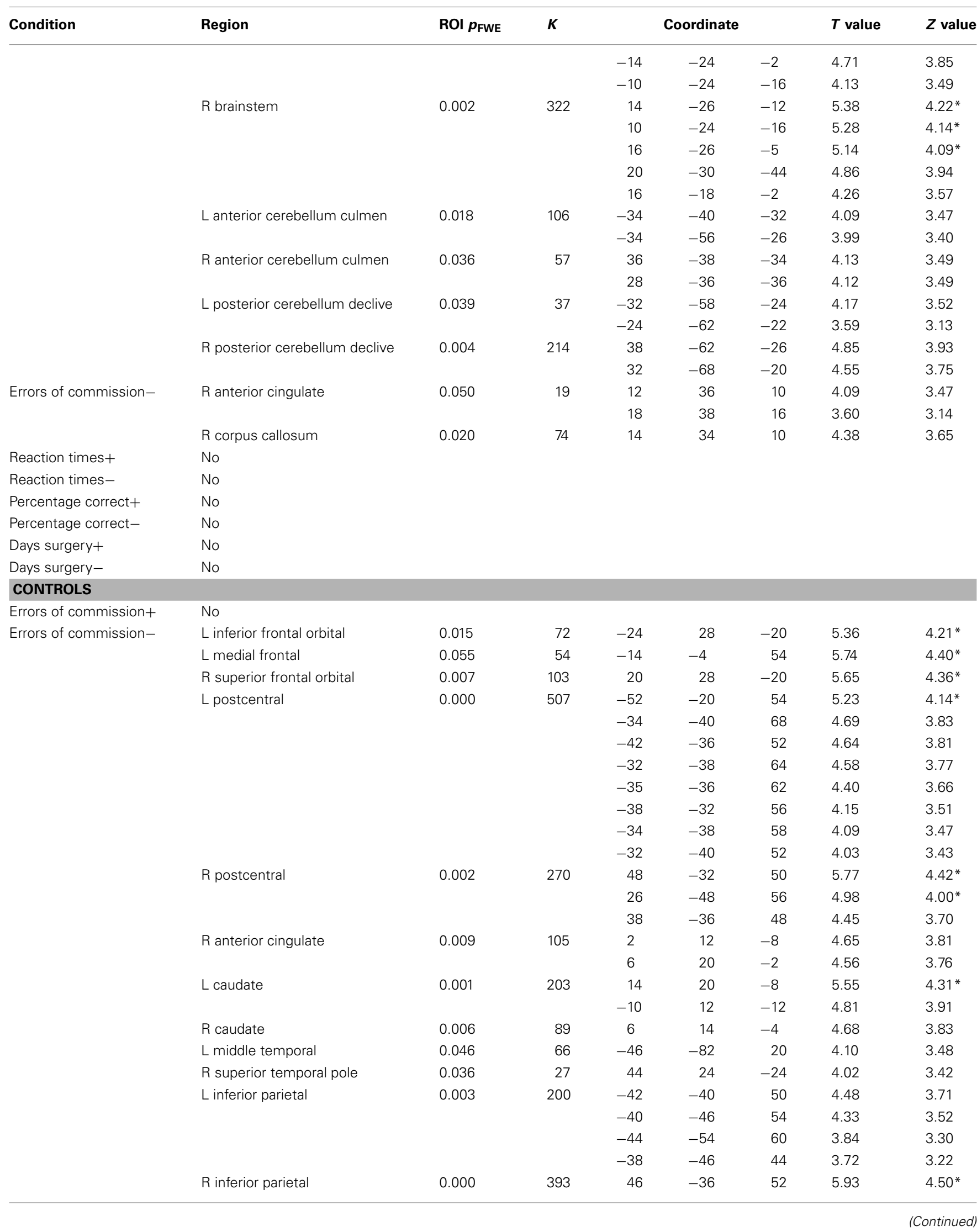




\begin{tabular}{|c|c|c|c|c|c|c|c|c|}
\hline \multirow[t]{2}{*}{ Condition } & \multirow[t]{2}{*}{ Region } & \multirow[t]{2}{*}{ ROI $p_{\mathrm{FWE}}$} & \multirow[t]{2}{*}{$\kappa$} & \multicolumn{3}{|c|}{ Coordinate } & \multirow{2}{*}{$\begin{array}{l}\boldsymbol{T} \text { value } \\
5.64\end{array}$} & \multirow{2}{*}{$\frac{Z \text { value }}{4.35^{*}}$} \\
\hline & & & & 36 & -44 & 46 & & \\
\hline & & & & 42 & -38 & 50 & 5.43 & $4.24^{*}$ \\
\hline & & & & 56 & -30 & 52 & 5.02 & $4.03^{*}$ \\
\hline & L middle occipital & 0.000 & 393 & -20 & -94 & 4 & 4.97 & $3.99 *$ \\
\hline & & & & -44 & -84 & 10 & 4.31 & 3.61 \\
\hline & & & & -18 & -102 & 10 & 4.30 & 3.60 \\
\hline & & & & -28 & -96 & 10 & 4.27 & 3.59 \\
\hline & & & & -18 & -100 & 4 & 4.24 & 3.56 \\
\hline & & & & -44 & -86 & 14 & 4.22 & 3.55 \\
\hline Reaction times+ & No & & & & & & & \\
\hline Reaction times- & No & & & & & & & \\
\hline \multirow[t]{4}{*}{ Percentage correct+ } & $L$ inferior frontal & 0.041 & 70 & -40 & 20 & -4 & 5.34 & $4.20 *$ \\
\hline & L superior orbital frontal & 0.028 & 33 & -12 & 50 & -20 & 4.61 & 3.79 \\
\hline & $L$ medial frontal & 0.011 & 51 & -8 & 48 & -8 & 4.06 & 3.45 \\
\hline & L parahippocampus & 0.021 & 42 & -14 & 4 & 22 & 5.02 & $4.02 *$ \\
\hline Percentage correct- & No & & & & & & & \\
\hline
\end{tabular}

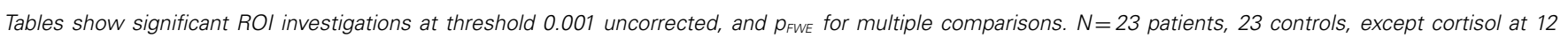
patients 15 controls. $R$, right; L, left; + , positive; -, negative; *indicates significance at Bonferroni adjustment for multiple comparisons.

to recruitment of this region to suppress motor responses in order to increase task accuracy. A well-studied neuro-modulatory system related to the prefrontal cortex, in order to adjust to specific tasks, is the dopaminergic input from the midbrain (particularly in the ventral tegmental area and substantia nigra; Durstewitz et al., 2000). Previous research has indicated that dopaminergic activity increases during working memory tasks (Schultz et al., 1993; Watanabe, 1996), which could help explain why patients also show increased midbrain activity compared to controls when completing this task, along with their increased frontal activity.

Of particular interest is the addition of "reaction time" as a covariate, which removed right midbrain differences between groups, and modified the results to reveal increased activity in bilateral thalamus and right superior occipital gyrus in patients compared to controls. Thalamic activations have been linked to performance in attention-demanding reaction time tasks (Kinomura et al., 1996). Considering the close proximity/overlap of the left inferior frontal operculum and insula coordinates, activity in this region was not affected by the addition of reaction time as a covariate. Also, depression and anxiety scores were significantly different between groups (although both in the mild range), yet only $\mathrm{BDI}$ scores modified group differences. The addition of BDI scores as a covariate adjusted the findings to uncover that there was no longer left inferior frontal operculum activity, but midbrain activations were maintained. Also notable, both days since surgery as well as errors of commission added as separate covariates completely eliminated any group differences observed in the no covariates analysis. At this stage of the research it is difficult to determine the relationship between each of these variables and their contribution to cognitive deficits in pre-chemotherapy BC patients. Overall, the addition of suspected confounding variables as covariates in the $t$-test analysis modifies existing group differences, thereby indicating that they are impacting cognition-related brain activity. These should be considered in baseline analyses of pre-chemotherapy patients as well as independently studied in order to better understand their mechanisms of action.

In an attempt to understand the results more thoroughly, analyses contrasting the active tasks with the rest condition were performed and revealed more additional group differences.

Patients showed significantly less widespread brain activation compared to controls when subtracting rest from either of the active tasks (either press for 2 back or press for O). (Patients did show significantly more activation than controls in some region, but this was a less substantial finding than the latter.) Such a distinction between groups perhaps suggests that reduced brain activity in patients in both these contrasts is due to increased neural activity at rest, lessened activation during both of the active task blocks or a combination of both. Overall, patients show less of a magnitude of activation between the rest condition and both of the active task conditions compared to controls. One study applied PET techniques to investigate glucose metabolism at rest in 
chemotherapy-treated patients compared to non-chemotherapy cancer patients (Baudino et al., 2011). Patients who had recently received larger doses of chemotherapy revealed lower glucose metabolic rates in bilateral prefrontal cortex, cerebellum, posterior medial regions, and limbic lobe compared to healthy controls and patients who had received smaller chemotherapy doses at an earlier time. (There were no differences between Late-low patients compared to those without chemotherapy-treatment.) This study indicates that patients treated with chemotherapy may have altered resting states, yet it seems to be transient in nature. The prevailing brain regions uncovered as significant in this PET study are among those found activated at a lesser extent in patients compared to controls in the current paper when rest is subtracted from the active task. Meanwhile there is some overlap in the frontal and limbic regions where patients inversely show larger activity compared to controls, as well as consistently in the thalamus, striatum, and brainstem. However, one must consider that the visuospatial task used in this paper had only 2 rest epochs of $24 \mathrm{~s}$ each. Such a small rest block could mean that participants were not truly at rest, possibly still dwelling on actions related to the preceding active task. Longer rest conditions or resting state fMRI to investigate the default network functioning in BC patients, both pre- and post-treatment are warranted and should be conducted in future studies. Additionally, investigations into the ability of patients to "sustain" active task brain activations compared to controls would be interesting. It is possible that the major finding concerning decreased magnitude of activity between active task and rest seen in patients could be a consequence of difficulties in sustaining active task attention. Patients may potentially be fluctuating between neural engagement during the active task and default brain activity over the course of the active task block themselves.

Further statistical exploration of the data included regressions analyses considering factors such as errors of commission, reaction time, percent correct, and days since surgery. While it is expected to find within-group variability in any study sample, the nature of heterogeneity revealed in the patient and control samples, respectively, is quite interesting. There were no significant relationships between brain activation and reaction time or days since surgery. However, regression analyses in patients along errors of commission revealed a positive relationship between the number of erroneous button presses during the task and the amount of neural activity in the insula, cingulate gyrus, hippocampus, parahippocampus, inferior temporal gyrus, thalamus, fusiform as well as brainstem and cerebellum. This is particularly interesting given the small number of commission errors committed by both groups of participants. Patients, who had slower response times than controls also made less of these mistakes, suggesting a compensation of speed for accuracy or a more cautious approach in the patient group. The only other pre-chemotherapy fMRI study of working memory to show significant differences between BC patients and controls also revealed slower reaction time and less accuracy in patients compared to controls. (Cimprich et al., 2010). Meanwhile, in the control population, there was increased widespread brain activity as less commission errors were being committed, mainly in frontal and parietal regions. There is little overlap between the activated regions found in patients and controls, again highlighting the differences in neural processing during visuospatial working memory between pre-treatment patients and controls along this variable.

Similarly, when considering percent correct scores, controls revealed an expected relationship between neural activity and performance, with more activation in frontal areas and parahippocampal gyrus related to better performance. Patients did not show this relationship, thereby not revealing within-group variability when considering this variable's impact on brain functioning. Therefore, it seems like patients do not show a typical linear relationship between task performance and increased brain activations as patients with task success show the same brain activity as those who committed many errors of omission. These withinsample regression results highlight the importance of including more than just analyses of group differences (such as $t$-test or factorial analysis) as these may wash out important subtle variations revealed in the individual groups. The opposite relationship between task commission errors and neural activity in patient and control populations indicates different within-group variability. As well, it is interesting to note that patients do not reveal a typical linear relationship between increased brain activity and higher task performance, as seen in controls. Such within-group relationships should additionally be systematically studied since it could yield a better understanding of post-chemotherapy sequelae and possible pre-disposition factors leading to more serious cognitive impairment.

To our knowledge, this study is the largest prospective fMRI investigation of brain activation patterns during visuospatial working memory of pre-chemotherapy cancer patients compared to individually matched controls. Some fMRI studies have not revealed functional pre-chemotherapy differences (Saykin et al., 2006) which may be due to smaller sample sizes and different task modalities. Possible limitations should be considered. First, the present study was performed on a 1.5-T scanner. As shown in a previous study (Krasnow et al., 2003), use of a 3-T scanner could uncover more activations, particularly in the frontal regions. Second, a smaller number of patients and controls, compared to the total sample, completed the diurnal cortisol and estrogen salivary sampling sessions. This number was restricted in patients due to practical constraints. While not a significant effect, pretreatment BC patients in this study revealed a flattened diurnal cortisol curve similar to that observed in post-treatment studies (Abercrombie et al., 2004; Spiegel et al., 2006). As well, it is known that successful working memory is affected by HPA activity (Lupien et al., 1999; Al'Absi et al., 2002; Oei et al., 2006). Therefore, future investigations of pre-treatment BC patients should consider cortisol assessments in a larger population. Lastly, it is difficult to separate working memory from other cognitive processes such as impulsivity and attention, which may also be impacted by factors related to BC, pre-chemotherapy. This was controlled as much as possible in the current block-design task, but perhaps an event related design could isolate these different processes further.

In conclusion, these results provide preliminary but compelling evidence of neurofunctional differences between BC patients and controls associated with visuospatial working memory, prior to chemotherapy. Additionally, they expose the importance of certain 
confounding variables, leading to the suggestion that these should be studied as primary variables of interest in future research. Also, the findings indicate a polar neurofunctional relationship between task performance and brain activity in patients compared to controls, as well as potential differences in the magnitude of brain activity between active task engagement and rest. These results have significant implications for future cancer and chemotherapy cognitive research, concerning both study designs and analyses. Understanding cancer patients prior to chemotherapy, and the contribution of confounding variables on baseline differences will lead to a more precise understanding of cognitive impairments related to chemotherapy-treatment itself. It will also assist with identifying pre-chemotherapy cognitive vulnerability, particularly in working memory, an essential capacity in this challenging time in their life. A new cancer diagnosis leads to many challenges

\section{REFERENCES}

Abercrombie, H. C., Giese-Davis, J., Sephton, S., Epel, E. S., Turner-Cobb, J. M., and Spiegel, D. (2004). Flattened cortisol rhythms in metastatic breast cancer patients. Psychoneuroendocrinology 29, 1082-1092.

Abraham, J., Haut, M. W., Moran, M. T., Filburn, S., Lemiuex, S., and Kuwabara, H. (2008). Adjuvant chemotherapy for breast cancer: effects on cerebral white matter seen in diffusion tensor imaging. Clin. Breast Cancer 8, 88-91.

Aguirre, G. K., Detre, J. A., Alsop, D. C., and D'Esposito, M. (1996). The parahippocampus subserves topographical learning in man. Cereb. Cortex 6, 823-829.

Ahles, T. A., Saykin, A. J., Furstenberg, C. T., Cole, B., Mott, L. A., Skalla, K., Whedon, M. B., Bivens, S., Mitchell, T., Greenberg, E. R., and Silberbarb, P. M. (2002). Neuropsychologic impact of standard-dose systemic chemotherapy in long-term survivors of breast cancer and lymphoma. J. Clin. Oncol. 20, 485-493.

Ahles, T. A., Tope, D. M., Furstenberg, C., Hann, D., and Mills, L. (1996). Psychologic and neuropsychologic impact of autologous bone marrow transplantation. J. Clin. Oncol. 14, 1457-1462.

Al'Absi, M., Hugdahl, K., and Lovallo, W. R. (2002). Adrenocortical stress responses and altered working memory performance. Psychophysiology 39, 95-99.

Alichniewicz, K. K., Nebl, H., Klünemann, H. H., and Greenlee, M. W. (2010). The neural correlates of visuo-spatial working memory in patients with amnestic mild cognitive impairment. Eur. Psychiatry 25, 765.

Army Individual Test Battery. (1944). Manual for Directions and Scoring. Washington, DC: War Department, Adjutant General's Office.
Aron, A. R., Behrens, T. E., Smith, S., Frank, M. J., and Poldrack, R. A. (2007). Triangulating a cognitive control network using diffusionweighted magnetic resonance imaging (MRI) and functional MRI. J. Neurosci. 27, 3743-3752.

Backhaus, J., Junghanns, K., and Hohagen, F. (2004). Sleep disturbances are correlated with decreased morning awakening salivary cortisol. Psychoneuroendocrinology 29, 1184-1191.

Baddeley, A. D. (2000). The episodic buffer: a new component of working memory? Trends Cogn. Sci. 4, 417-423.

Baddeley, A. D., and Hitch, G. J. (1974). "Working memory," in Recent Advances in Learning and Motivation, ed. G. A. Bower (New York: Academic Press), 47-89.

Barch, D. M., Braver, T. S., Nystrom, L. E., Forman, S. D., Noll, D. C., and Cohen, J. D. (1997). Dissociating working memory from task difficulty in human prefrontal cortex. Neuropsychologia 35, 1373-1380.

Baudino, B., D’Agata, F., Castellano, G., Caroppo, P., Cauda, S., Parente, A., Manfredi, M., Geda, E., Orsi, L., Cauda, S., Castelli, L., Sacco, K., Ardito, R., Tora, R., and Bisi, G. (2011). Chemotherapy effects on brain glucose metabolism at rest. Nat. Precedings. doi: hdl:10101/npre.2011.5637.1

Beck, A. T., and Steer, R. A. (1990). Beck Anxiety Inventory Manual. San Antonio: Psychological Corporation.

Beck, A. T., Steer, R. A., and Brown, G. K. (1996). Beck Depression Inventory2nd Edn Manual. San Antonio: Psychological Corporation.

Behl, C. (2002). Oestrogen as a neuroprotective hormone. Nat. Rev. Neurosci. 3, 433-442.

for patients, both biological (cytokine activity, anesthesia) and personal (stress, anxiety, depression) which deserve to be systematically studied. Exposing vulnerability prior to chemotherapy is important since this is a time when many decisions must be made. With processes related to executive functioning affected pre-chemotherapy, the decision process itself is somewhat compromised, which adds to the already demanding situation. If these cancer patients are cognitively impacted during this time, it is also important for the medical professionals, family, and friends involved in decision making with the patient to be aware of these deficits.

\section{ACKNOWLEDGMENTS}

The Canadian Breast Cancer Foundation has funded this project. Their contribution was solely financial support.

Bender, C. M., Sereika, S. M., Berga, S. L., Vogel, V. G., Brufksy, A. M., Paraska, K. K., and Ryan, C. M. (2006). Cognitive impairment associated with adjuvant therapy in breast cancer. Psychooncology 15, 422-430.

Benedict, R. H. B. (1997). Brief Visuospatial Memory Test - Revised. Odessa, FL: Psychological Assessment Resources, Inc.

Benton, A. L., Hamsher, K., and Sivan, A. B. (1994). Multilingual Aphasia Examination, 3rd Edn. Iowa City, IA: AJA.

Berglund, G., Bolund, C., Fornander, T., Rutqvist, L. E., and Sjoden, P. (1991). Late effects of adjuvant chemotherapy on quality of life among breast cancer patients. Eur. J. Cancer 27, 1075-1081.

Boone, K. B., Miller, B. L., Lesser, I. M., Hill, E., and D'Elia, L. (1990). Performance on frontal lobe tests in healthy, older individuals. Dev. Neuropsychol. 6, 215-223.

Brandt, J., and Benedict, R. H. B. (2001). Hopkins Verbal Learning Test - Revised. Professional Manual. Lutz, FL: Psychological Assessment Resources, Inc.

Bremner, J. D., Southwick, S. M., and Charney, D. S. (1999). "The neurobiology of posttraumatic stress disorder: an integration of animal and human research," in Posttraumatic Stress Disorder: A Comprehensive Text, eds P. A. Saigh and D. Bremner (New York: Allyn and Bacon), 103-143.

Brezden, C., Phillips, K. A., Bunston, T., and Tannock, I. F. (2000). Cognitive function in breast cancer patients receiving adjuvant chemotherapy. J. Clin. Oncol. 18, 2695-2701.

Brown, M. S., Stemmer, S. M., Simon, J. H., Stears, J. C., Jones, R. B., Cagnoni, P. J., and Sheeder, J. L. (1998). White matter disease induced by high-dose chemotherapy: longitudinal study with $\mathrm{MR}$ imaging and proton spectroscopy. AJNR Am. J. Neuroradiol. 19, 217-221.

Buch, E. R., Mars, R. B., Boorman, E. D., and Rushworth, M. F. (2010). A network centered on ventral premotor cortex exerts both facilitatory and inhibitory control over primary motor cortex during action reprogramming. J. Neurosci. 30, 1395-1401.

Carlson, S., Martinkauppi, S., Rama, P., Salli, E., Korvenoja, A., and Aronen, H. J. (1998). Distribution of cortical activation during visuospatial n-back tasks as revealed by functional magnetic resonance imaging. Cereb. Cortex 8, 743-752.

Castellon, S. A., Ganz, P. A., Bower, J. E., Petersen, L., Abraham, L., and Greendale, G. A. (2004). Neurocognitive performance in breast cancer survivors exposed to adjuvant chemotherapy and tamoxifen. J. Clin. Exp. Neuropsychol. 26, 955-969.

Cimprich, B., Reuter-Lorenz, P., Nelson, J., Clark, P. M., Therrien, B., Normolle, D., Berman, M. G., Hayes, D. F., Noll, D. C., Pelletier, S., and Welsh, R. C. (2010). Prechemotherapy alterations in brain function in women with breast cancer. J. Clin. Exp. Neuropsychol. 32, 324-331.

Correa, D. D., and Ahles, T. A. (2007). Cognitive adverse effects of chemotherapy in breast cancer patients. Curr. Opin. Support. Palliat. Care 1, 57-62.

de Ruiter, M. B., Reneman, L., Boogerd, W., Veltman, D. J., van Dam, F. S., Nederveen, A. J., Boven, E., and Schagen, S. B. (2011). Cerebral hyporesponsiveness and cognitive impairment 10 years after chemotherapy for breast cancer. Hum. Brain Mapp. 32, 1206-1219. 
Denkla, M. B. (1993). "Measurement of executive functioning," in Frames of Reference for the Assessment of Learning Disabilities: New Views on Measurement Issues, ed. G. R. Lyon (Baltimore: Paul Brookes), 117-142.

Deprez, S., Amant, F., Yigit, R., Porke, K., Verhoeven, J., Van den Stock, J., Smeets, A., Christiaens, M. R., Leemans, A., Van Hecke, W., Vandenberghe, J., Vandenbulcke, M., and Sunaert, S. (2011). Chemotherapyinduced structural changes in cerebral white matter and its correlation with impaired cognitive functioning in breast cancer patients. Hum. Brain Mapp. 32, 480-493.

Desmond, J. E., Chen, S. H., DeRosa, E., Pryor, M. R., Pfefferbaum, A., and Sullivan, E. V. (2003). Increased frontocerebellar activation in alcoholics during verbal working memory: an fMRI study. Neuroimage 19, 1510-1520.

Dijkstra, J. B., Houx, P. J., and Jolles, J. (1999). Cognition after major surgery in the elderly: test performance and complaints. $\mathrm{Br}$. J. Anaesth. 82, 867-874.

Dodds, C., and Allison, J. (1998). Postoperative cognitive deficit in the elderly surgical patient. $\mathrm{Br}$. J. Anaesth. 81, 449-462.

Durstewitz, D., Seamans, J. K., and Sejnowski, T. J. (2000). Neurocomputational models of working memory. Nat. Neurosci. 3(Suppl.), 1184-1191.

Ferguson, R. J., McDonald, B. C., Saykin, A. J., and Ahles, T. A. (2007). Brain structure and function differences in monozygotic twins: possible effects of breast cancer chemotherapy. J. Clin. Oncol. 25, 3866-3870.

Friston, K. J., Ashburner, J., Poline, J. B., Frith, C. D., Heather, J. D., and Frackowiak, R. S. J. (1995). Spatial realignment and normalization of images. Hum. Brain Mapp. 2, 165-189.

Fuster, J. M. (1997). The PFC: Anatomy, Physiology and the Neuropsychology of the Frontal Lobe. New York: Raven Press, 410

Glikmann-Johnston, Y., Saling, M. M., Chen, J., Cooper, K. A., Beare, R. J., and Reutens, D. C. (2008). Structural and functional correlates of unilateral mesial temporal lobe spatial memory impairment. Brain 131, 3006-3018.

Goodwin, P. J., Ennis, M., Pritchard, K. I., Trudeau, M., and Hood, N. (1999). Risk of menopause during the first year after breast cancer diagnosis. J. Clin. Oncol. 17, 2365-2370.

Gottschalk, L. A., Holcombe, R. F., Jackson, D., and Bechtel, R. J. (2003). The effects of anticancer chemotherapeutic drugs on cognitive function and other neuropsychiatric dimensions in breast cancer patients. Methods Find. Exp. Clin. Pharmacol. 25, 117-122.

Gronwall, D. M. A. (1997). Paced auditory serial addition task. A measure of recovery from concussion. Percept. Mot. Skills 44, 367-373.

Gualtieri, C. T., and Johnson, L. G. (2006). Reliability and validity of a computerized neurocognitive test battery, CNS vital signs. Arch. Clin. Neuropsychol. 21, 623-643.

Haberecht, F., Menon, V., Warsofsky, I. S., White, C. D., Dyer-Friedman, J., Glover, G. H., Neely, E. K., and Reiss, A. L. (2001). Functional neuroanatomy of visuo-spatial working memory in turner syndrome. Hum. Brain Mapp. 14, 96-107.

Haley, A. P., Gunstad, J., Cohen, R. A., Jerskey, B. A., Mulligan, R. C., and Sweet, L. H. (2008). Neural correlates of visuospatial working memory in healthy young adults at risk for hypertension. Brain Imaging Behav. 2, 192-199.

Hurria, A., Rosen, C., Hudis, C., Zuckerman, E., Panageas, K. S., Lachs, M. S., Witmer, S., van Gorp, W. G., Fornier, M., D’Andrea, G., Moasser, M., Dang, C., Van Poznak, C., Hurria, A., and Holland, J. (2006). Cognitive function of older patients receiving adjuvant chemotherapy for breast cancer: a pilot prospective longitudinal study. J. Am. Geriatr. Soc. 54, 925-931.

Inagaki, M., Yoshikawa, E., Matsuoka, Y., Sugawara, Y., Nakano, T., Akechi, T., Wada, N., Imoto, S., Murakami, K., and Uchitomi, Y. (2007). Smaller regional volumes of brain gray and white matter demonstrated in breast cancer survivors exposed to adjuvant chemotherapy. Cancer 109, 146-156.

Jenkins, V., Shilling, V., Deutsch, G., Bloomfield, D., Morris, R., Allan, S., Bishop, H., Hodson, N., Mitra, S., Sadler, G., Shah, E., Whitehead, S., and Winstanley, J. (2006). A 3year prospective study of the effects of adjuvant treatments on cognition in women with early stage breast cancer. Br. J. Cancer 94, 828-834.

Jonides, J., Smith, E. E., Koeppe, R. A., Awh, E., Minoshima, S., and Mintun, M. A. (1993). Spatial working memory in humans as revealed by PET. Nature 363, 623-625.

Kesler, S. R., Bennett, F. C., Mahaffey, M. L., and Spiegel, D. (2009). Regional brain activation during verbal declarative memory in metastatic breast cancer. Clin. Cancer Res. 15, 6665-6673.

Kinomura, S., Larsson, J., Gulyás, B., and Roland, P. E. (1996). Activation by attention of the human reticular formation and thalamic intralaminar nuclei. Science 26, 512-515.

Kirschbaum, C., and Hellhammer, D. H. (1994). Salivary cortisol in psychoneuroendocrine research recent developments and applications. Psychoneuroendocrinology 19, 313-333.

Krampen, G. (1991). Fragebogen $z u$ Kompetenz und Kontrollueberzeugungen (FKK). Göttingen: Hogrefe.

Krasnow, B., Tamm, L., Greicius, M. D. Yang, T. T., Glover, G. H., Reiss, A. L., and Menon, V. (2003). Comparison of fMRI activation at 3 and 1.5 $\mathrm{T}$ during perceptual, cognitive, and affective processing. Neuroimage 18, 813-826.

Kreukels, B. P., Hamburger, H. L., de Ruiter, M. B., van Dam, F. S., Ridderinkhof, K. R., Boogerd, W., and Schagen, S. B. (2008). ERP amplitude and latency in breast cancer survivors treated with adjuvant chemotherapy. Clin. Neurophysiol. 119, 533-541.

Kreukels, B. P., Schagen, S. B., Ridderinkhof, K. R., Boogerd, W., Hamburger, H. L., Muller, M. J., and van Dam, F. S. (2006). Effects of high-dose and conventional-dose adjuvant chemotherapy on longterm cognitive sequelae in patients with breast cancer: an electrophysiologic study. Clin. Breast Cancer 7, 67-78.

Kreukels, B. P., Schagen, S. B., Ridderinkhof, K. R., Boogerd, W., Hamburger, H. L., and van Dam, F. S. (2005). Electrophysiological correlates of information processing in breast-cancer patients treated with adjuvant chemotherapy. Breast Cancer Res. Treat. 94, 53-61.

Lord, C., Buss, C., Lupien, S. J., and Pruessner, J. C. (2008). Hippocampal volumes are larger in postmenopausal women using estrogen therapy compared to past users, never users and men: a possible window of opportunity effect. Neurobiol. Aging 29, 95-101.

Lower, E. E., Blau, R., Gazder, P., and Tummala, R. (1999). The risk of premature menopause induced by chemotherapy for early breast cancer. J. Womens Health Gend. Based Med. 8, 949-954.

Lupien, S. J., Gillin, C. J., and Hauger, R. L. (1999). Working memory is more sensitive than declarative memory to the acute effects of corticosteroids: a dose-response study in humans. Behav. Neurosci. 113, 420-430.

MacHale, S. M., Cavanagh, J. T. O., Bennie, J., Carroll, S., Goodwin, G. M., and Lawrie, S. M. (1998). Diurnal variation of adrenocortical activity in chronic fatigue syndrome. $\mathrm{Neu}$ ropsychobiology 38, 213-217.

Maguire, E. A., Frith, C. D., Burgess, N., Donnett, J. G., and O'Keefe, J. (1998). Knowing where things are: parahippocampal involvement in encoding object location in virtual large-scale space. J. Cogn. Neurosci. 10, 61-76.

Maguire, E. A., Mummery, C. J., and Buchel, C. (2000). Patterns of hippocampal-cortical interaction dissociate temporal lobe memory subsystems. Hippocampus 10, 475-482.

Maier, S. F., and Watkins, L. R. (2003). Immune-to-central nervous system communication and its role in modulating pain and cognition: implications for cancer and cancer treatment. Brain Behav. Immun. 17, 125-131.

Mar Fan, H. G., Houédé-Tchen, N., Yi, Q. L., Chemerynsky, I., Downie, F. P., Sabate, K., and Tannock, I. F. (2005). Fatigue, menopausal symptoms, and cognitive function in women after adjuvant chemotherapy for breast cancer: 1- and 2-year follow-up of a prospective controlled study. J. Clin. Oncol. 31, 8025-8032.

McDonald, B. C., Conroy, S. K., Ahles, T. A., West, J. D., and Saykin, A. J. (2010). Gray matter reduction associated with systemic chemotherapy for breast cancer: a prospective MRI study. Breast Cancer Res. Treat. 123, 819-828.

McEwen, B. (2002). Estrogen actions throughout the brain. Recent Prog. Horm. Res. 57, 357-384.

McEwen, B. S. (1999). Stress and the aging hippocampus. Front. Neuroendocrinol. 20, 49-70.

McEwen, B. S., and Magarinos, M. (1997). Stress effects on morphology and function of the hippocampus. Ann. N. Y. Acad. Sci. 821, 271-284.

Monk, T. G., Weldon, B. C., Garvan, C. W., Dede, D. E., van der Aa, M. T., Heilman, K. M., and Gravenstein, J. S. (2008). Predictors of cognitive dysfunction after major noncardiac surgery. Anesthesiology 108, 18-30.

Monteleone, P., and Maj, M. (2009). Circadian rhythm disturbances in depression: implications for reatment and quality of remission. Medicographia 31, 132-139.

Noal, S., Levy, C., Hardouin, A., Rieux, C., Heutte, N., Ségura, C., Collet, F., Allouache, D., Swisters, O., Delcambre, C., Delozier, T., HenryAmar, M., and Joly, F. (2010). Oneyear longitudinal study of fatigue, cognitive functions, and quality of life after adjuvant radiotherapy for breast cancer. Int. J. Radiat. Oncol. Biol. Phys. 81, 795-803. 
Oei, N. Y. L., Everaerd, W. T. A. M., Elzinga, B. M., van Well, S. M., and Bermond, B. (2006). Psychosocial stress impairs working memory at high loads: an association with cortisol levels and memory retrieval. Stress 9, 133-141.

Ploner, C. J., Gaymard, B. M., Rivaud-Pechoux, S., Baulac, M., Clemenceau, S., Severine, S., and Pierrot-Deseilligny, C. (2000). Lesions affecting the parahippocampal cortex yield spatial memory deficits in humans. Cereb. Cortex 10, 1211-1216.

Pruessner, J. C., Baldwin, M. W., Dedovic, K., Renwick, R., Mahani, N. K., Lord, C., Meaney, M., and Lupien, S. (2005). Self-esteem, locus of control, hippocampal volume, and cortisol regulation in young and old adulthood. Neuroimage 28, 815-826.

Pruessner, J. C., Hellhammer, D. H., and Kirschbaum, C. (1999). Low self-esteem, induced failure and the adrenocortical stress response. Pers. Individ. Dif. 27, 477-489.

Pruessner, J. C., Kirschbaum, C., Meinlschmid, G., and Hellhammer, D. H. (2003). Two formulas for the computation of the area under the curve represent measures of total hormone concentration versus time-dependent change. Psychoneuroendocrinology 28, 916-931.

Pruessner, J. C., Wolf, O. T., Hellhammer, D. H., Buske-Kirschbaum, A. B., vonAuer, K., Jobst, S., Kaspers, F., and Kirschbaum, C. (1997). Free cortisol levels after awakening: a reliable biological marker for the assessment of adrenocortical activity. Life Sci. 61, 2539-2549.

Raffa, R. B., and Tallarida, R. J. (2010). Effects on the visual system might contribute to some of the cognitive deficits of cancer chemotherapy-induced "chemofog." J. Clin. Pharm. Ther. 35, 249-255.

Rosenberg, M. (1985). "Self-concept and psychological well-being in adolescence," in The Development of the Self, ed. R. L. Leahy (Orlando, FL: The Academic Press), 205-246.

Sapolsky, R. (1985). A possible mechanism for glucocorticoid toxicity in the hippocampus: increased vulnerability of neurons to metabolic insults. J. Neurosci. 5, 1228-1232.

Saykin, A. J., Ahles, T. A., and Schoenfeld, J. D. (2003). Gray matter reduction on voxel-based morphometry in chemotherapy-treated cancer survivors. J. Int. Neuropsyhol. Soc. 9, 246.

Saykin, A. J., McDonald, B. C., Ahles, T., Chesnut, L. A., Wang, P. J., Fursten- berg, C. T., Horrigan, S. A., and Mamourian, A. C. (2006). "Altered brain activation following systemic chemotherapy for breast cancer: interim analysis from a prospective fMRI study," in Abstract presented at 34th Annual Meeting of the International Neuropsychological Society, Boston.

Schagen, S. B., Muller, M. J., Boogerd, W., Rosenbrand, R. M., van Rhijn, D., Rodenhuis, S., and van Dam, F. S. A. M. (2002). Late effects of adjuvant chemotherapy on cognitive function: a follow-up study in breast cancer patients. Ann. Oncol. 132, 1387-1397.

Schagen, S. B., van Dam, F., Muller, M. J., Boogerd, W., Lindeboom, J., and Bruning, P. F. (1999). Cognitive deficits after postoperative adjuvant chemotherapy for breast carcinoma. Cancer 85, 640-650.

Scherwath, A., Mehnart, A., Schleimer, B., Schirmer, L., Fehlauer, F., Kreienberg, R., Metzner, B., Thiel, E., Zander, A. R., and Schulz-Kindermann, F. (2006). Neuropsychological function in high-risk breast cancer survivors after stem-cell supported high-dose therapy versus standarddose chemotherapy: evaluation of long-term treatment effects. Ann. Oncol. 17, 415-423.

Schultz, W., Apicella, P., and Ljungberg, T. (1993). Responses of monkey dopamine neurons to reward and conditioned stimuli during successive steps of learning a delayed response task. J. Neurosci. 13, 900-913.

Servaes, P., Verhagen, C., and Bleijenberg, G. (2002). Relations between fatigue, neuropsychological functioning, and physical activity after treatment for breast carcinoma: daily self-report and objective behavior. Cancer 95, 2017-2026.

Shackman, A. J., Sarinopoulos, I., Maxwell, J. S., Pizzagalli, D. A., Lavric, A., and Davidson, R. J. (2006). Anxiety selectively disrupts visuospatial working memory. Emotion 6, 40-61.

Shilling, V., Jenkins, V., Morris, R., Deutsch, G., and Bloomfield, D. (2005). The effects of adjuvant chemotherapy on cognition in women with breast cancer - preliminary results of an observational longitudinal study. Breast 14, 142-150.

Shipman, S. L., and Astur, R. S. (2008). Factors affecting the hippocampal BOLD response during spatial memory. Behav. Brain Res. 187, 433-441.

Shughrue, P. J., and Merchenthaler, I. (2000). Estrogen is more than just a "sex hormone": novel sites for estrogen action in the hippocampus and cerebral cortex. Front. Neuroendocrinol. 21, 95-101.

Silverman, D. H. S., Dy, C. J., Castellon, S. A., Lai, J., Pio, B. S., Abraham, L. Waddell, K., Petersen, L., Phelps, M. E., and Ganz, P. A. (2007). Altered frontocortical, cerebellar, and basal ganglia activity in adjuvant-treated breast cancer survivors 5-10 years after chemotherapy. Breast Cancer Res. Treat. 103, 303-311.

Smith, A. M., Fried, P. A., Hogan, M. J., and Cameron, I. (2006). Effects of prenatal marijuana on visuospatial working memory: an fMRI study in young adults. Neurotoxicol. Teratol. 28, 286-295.

Smith, A. M., Longo, C. A., Fried, P. A., Hogan, M. J., and Cameron, I. (2010). Effects of marijuana on visuospatial working memory: an fMRI study in young adults. Psychopharmacology (Berl.) 210, 429-438.

Smith, E. E., Jonides, J., and Koeppe, R. A. (1996). Dissociating verbal and spatial working memory using PET. Cereb. Cortex 6, 11-20.

Spiegel, D., Giese-Davis, J., Taylor, C. B., and Kraemer, H. (2006). Stress sensitivity in metastatic breast cancer: analysis of hypothalamic-pituitaryadrenal axis function. Psychoneuroendocrinology 31, 1231-1244.

Starkman, M. N., Gebarski, S. S., Berent, S., and Schteingart, D. E. (1992). Hippocampal formation volume, memory dysfunction, and cortisol levels in patients with Cushing's syndrome. Biol. Psychiatry 32, 756-765.

Stewart, A., Collins, B., MacKenzie, J. Tomiak, E., Verma, S., and Bielajew, C. (2007). The cognitive effects of adjuvant chemotherapy in early stage breast cancer: a prospective study. Psychoongology 17, 1-9.

Tchen, N., Juffs, H. G., Downie, F. P., Yi, Q. L., Hu, H., Chemerynsky, I., Clemons, M., Crump, M., Goss, P. E. Warr, D., Tweedale, M. E., and Tannock, I. F. (2003). Cognitive function, fatigue, and menopausal symptoms in women receiving adjuvant chemotherapy for breast cancer. $J$. Clin. Oncol. 21, 4175-4183.

Tuxen, M. K., and Werner, H. S. (1994). Neurotoxicity secondary to antineoplastic drugs. Cancer Treat. Rev. 20, 191-214.

van Dam, F., Schagen, S. B., Muller, M. J., Boogerd, W., Wall, E. v. d., Droogleever Fortuyn, M. E., and Rodenhuis, S. (1998). Impairment of cognitive function in women receiving adjuvant treatment for high-risk breast cancer: high-dose versus standard-dose chemotherapy. J. Natl. Cancer Inst. 90, 210-218.
Vardy, J., Booth, C., Pond, G. R., Zhang, H., Galica, J., Dhillon, H., Clarke, S. J., and Tannock, I. F. (2007). "Cytokine levels in patients with colorectal cancer and breast cancer and their relationship to fatigue and cognitive function," in Abstract Presented at the American Society of Clinical Oncology-Poster, Patient and Survivor Care, Chicago, IL, 9070.

Wagner, L. I., Sweet, J. J., Butt, Z., Desai, J., Beaumont, J., Havlin, K. A., Sabatino, T., and Cella, D. (2006). "Cognitive impairment associated with chemotherapy for breast cancer: an exploratory case-control study," in Abstract Presented at American Society of Clinical OncologyClinical Science Symposium, Cognitive Impairment in Cancer Survivors, Atlanta, GA, 8501.

Watanabe, M. (1996). Reward expectancy in primate prefrontal neurons. Nature 382, 629-632.

Wechsler, D. (1997). Wechsler Adult Intelligence Scale, 3rd Edn. San Antonio: Harcourt, Brace, and Co.

Wefel, J. S., Lenzi, R., Theriault, R. L., Davis, R. N., and Meyers, C. A. (2004). The cognitive sequelae of standard-dose adjuvant chemotherapy in women with breast carcinoma. Cancer 100, 2292-2299.

Wieneke, M. H., and Dienst, E. R. (1995). Neuropsychological assessment of cognitive functioning following chemotherapy for breast cancer. Psychooncology 4, 61-66.

Conflict of Interest Statement: The authors declare that the research was conducted in the absence of any commercial or financial relationships that could be construed as a potential conflict of interest.

Received: 23 August 2011; paper pending published: 26 September 2011; accepted: 10 October 2011; published online: 01 November 2011.

Citation: Scherling C, Collins B, MacKenzie J, Bielajew $C$ and Smith A (2011) Pre-chemotherapy differences in visuospatial working memory in breast cancer patients compared to controls: an fMRI study. Front. Hum. Neurosci. 5:122. doi: 10.3389/fnhum.2011.00122 Copyright (c) 2011 Scherling, Collins, MacKenzie, Bielajew and Smith. This is an open-access article subject to a nonexclusive license between the authors and Frontiers Media SA, which permits use, distribution and reproduction in other forums, provided the original authors and source are credited and other Frontiers conditions are complied with. 


\section{APPENDIX}

Table A1 | Significant fixed-effects for patients and controls for the 2 back-0 contrast.

\begin{tabular}{|c|c|c|c|c|c|c|c|c|}
\hline $\begin{array}{l}\text { Condition } \\
\text { Patients }\end{array}$ & $\begin{array}{l}\boldsymbol{p}_{\text {FWE }} \\
0.000\end{array}$ & $\begin{array}{l}\boldsymbol{K} \\
369\end{array}$ & $\begin{array}{l}\text { Region } \\
\text { L inferior frontal gyrus }\end{array}$ & \multicolumn{3}{|c|}{ Coord. } & $\begin{array}{l}\boldsymbol{T} \text { value } \\
6.62\end{array}$ & $\begin{array}{l}Z \text { value } \\
5.48\end{array}$ \\
\hline & & & & -50 & 12 & 20 & 5.85 & 5.01 \\
\hline & & & & -50 & 10 & 24 & 5.56 & 4.81 \\
\hline & & & & -42 & 8 & 22 & 5.51 & 4.78 \\
\hline & & & & -52 & 16 & 24 & 5.44 & 4.73 \\
\hline & & & $\mathrm{L}$ insula & -46 & 10 & 14 & 5.85 & 5.01 \\
\hline & 0.000 & 400 & $\mathrm{R}$ inferior frontal trigeminal gyrus & 44 & 28 & 20 & 6.34 & 5.32 \\
\hline & & & $\mathrm{R}$ middle frontal gyrus & 46 & 38 & 24 & 7.51 & 5.99 \\
\hline & & & & 44 & 34 & 26 & 6.77 & 5.57 \\
\hline & & & & 48 & 34 & 38 & 6.62 & 5.49 \\
\hline & & & & 42 & 32 & 22 & 6.32 & 5.30 \\
\hline & 0.009 & 23 & $\mathrm{R}$ inferior frontal gyrus & 50 & 18 & 2 & 5.68 & 4.89 \\
\hline & 0.000 & 441 & $L$ middle frontal gyrus & -28 & 4 & 54 & 8.15 & 6.33 \\
\hline & & & & -30 & 2 & 50 & 7.85 & 6.17 \\
\hline & & & & -24 & 2 & 56 & 7.42 & 5.94 \\
\hline & & & & -26 & 4 & 60 & 7.34 & 5.90 \\
\hline & & & $\mathrm{L}$ precentral gyrus & -40 & -2 & 46 & 5.23 & 4.59 \\
\hline & 0.000 & 219 & $\mathrm{R}$ middle frontal gyrus & 32 & 60 & 8 & 6.56 & 5.45 \\
\hline & & & & 32 & 52 & 14 & 5.65 & 4.87 \\
\hline & 0.000 & 333 & $\mathrm{R}$ middle frontal gyrus & 30 & 22 & 52 & 6.21 & 5.24 \\
\hline & & & & 38 & 22 & 50 & 6.03 & 5.12 \\
\hline & & & & 32 & 16 & 52 & 5.98 & 5.09 \\
\hline & & & $\mathrm{R}$ superior medial frontal gyrus & 6 & 30 & 44 & 8.44 & 6.47 \\
\hline & 0.022 & 8 & $L$ inferior frontal gyrus & -30 & 22 & -4 & 5.42 & 4.72 \\
\hline & 0.030 & 4 & $\mathrm{R}$ insula & 30 & 24 & 0 & 5.39 & 4.69 \\
\hline & 0.003 & 47 & $\mathrm{R}$ inferior temporal gyrus & 58 & -46 & -16 & 5.14 & 4.53 \\
\hline & & & $\mathrm{R}$ middle temporal gyrus & 60 & -44 & -8 & 5.55 & 4.80 \\
\hline & 0.023 & 7 & $\mathrm{R}$ inferior temporal gyrus & 54 & -48 & -16 & 5.25 & 4.60 \\
\hline & 0.000 & 1367 & $\mathrm{R}$ inferior parietal lobule & 36 & -64 & 42 & 7.81 & 6.15 \\
\hline & & & & 40 & -64 & 46 & 7.75 & 6.12 \\
\hline & & & & 40 & -62 & 38 & 7.20 & 5.82 \\
\hline & & & & 36 & -54 & 40 & 6.89 & 5.64 \\
\hline & & & & 42 & -42 & 36 & 6.19 & 5.22 \\
\hline & & & & 46 & -50 & 34 & 6.14 & 5.19 \\
\hline & & & & 42 & -56 & 38 & 6.07 & 5.14 \\
\hline & & & L superior parietal lobule & -12 & -66 & 58 & 6.58 & 5.46 \\
\hline & & & & -20 & -72 & 56 & 6.24 & 5.26 \\
\hline & & & R superior parietal lobule & 36 & -68 & 44 & 7.71 & 6.10 \\
\hline & & & & 14 & -74 & 58 & 6.82 & 5.60 \\
\hline
\end{tabular}


Table A1 | Continued

\begin{tabular}{|c|c|c|c|c|c|c|c|c|}
\hline Condition & $p_{\mathrm{FWE}}$ & $\kappa$ & $\begin{array}{l}\text { Region } \\
\text { R supramarginal gyrus }\end{array}$ & \multicolumn{3}{|c|}{ Coord. } & $\begin{array}{l}\boldsymbol{T} \text { value } \\
6.99\end{array}$ & $\begin{array}{l}Z \text { value } \\
5.70\end{array}$ \\
\hline & & & & 40 & -54 & 42 & 6.35 & 5.32 \\
\hline & & & & 50 & -48 & 32 & 5.29 & 4.63 \\
\hline & & & R superior occipital & 30 & -64 & 40 & 7.47 & 5.97 \\
\hline & & & $L$ precuneus & -14 & -72 & 58 & 6.96 & 5.68 \\
\hline & & & R precuneus & 8 & -66 & 58 & 7.34 & 5.90 \\
\hline & & & & 30 & -68 & 42 & 7.22 & 5.84 \\
\hline & & & & 10 & -70 & 58 & 7.21 & 5.83 \\
\hline & 0.000 & 112 & $\mathrm{R}$ inferior parietal gyrus & 44 & -44 & 56 & 6.14 & 5.19 \\
\hline & 0.001 & 80 & L supramarginal gyrus & -38 & -46 & 36 & 6.24 & 5.25 \\
\hline & 0.030 & 4 & $\mathrm{R}$ cuneus & 26 & -84 & 20 & 5.25 & 4.60 \\
\hline \multirow{29}{*}{ Controls } & & & L precentral & -48 & 8 & 34 & 7.17 & 5.81 \\
\hline & & & & -42 & 6 & 34 & 6.82 & 5.60 \\
\hline & & & L middle frontal gyrus & -42 & 24 & 30 & 9.90 & 7.14 \\
\hline & & & & -28 & 4 & 54 & 9.25 & 6.86 \\
\hline & & & & -24 & 4 & 56 & 8.88 & 6.69 \\
\hline & & & & -54 & 20 & 36 & 7.19 & 5.81 \\
\hline & & & $\mathrm{R}$ middle frontal gyrus & 44 & 38 & 24 & 10.92 & 7.56 \\
\hline & & & & 44 & 30 & 35 & 9.15 & 6.81 \\
\hline & & & & 44 & 24 & 35 & 8.65 & 6.58 \\
\hline & & & & 35 & 8 & 54 & 8.48 & 6.50 \\
\hline & & & & 35 & 50 & 10 & 8.24 & 6.37 \\
\hline & & & & 50 & 14 & 46 & 7.24 & 5.84 \\
\hline & & & & 32 & 16 & 52 & 7.07 & 5.75 \\
\hline & & & & 40 & 20 & 48 & 6.59 & 5.47 \\
\hline & & & & 22 & 10 & 66 & 6.56 & 5.45 \\
\hline & & & $L$ medial frontal gyrus & -4 & 20 & 48 & 9.38 & 6.92 \\
\hline & & & $\mathrm{R}$ medial frontal gyrus & 6 & 22 & 50 & 10.56 & 7.42 \\
\hline & & & & 8 & 24 & 46 & 10.46 & 7.38 \\
\hline & & & R superior frontal gyrus & 24 & 8 & 54 & 6.98 & 5.70 \\
\hline & & & & 26 & 0 & 52 & 6.56 & 5.45 \\
\hline & 0.001 & 70 & $L$ insula & -34 & 22 & 0 & 6.15 & 5.19 \\
\hline & 0.000 & 5761 & $L$ inferior parietal lobule & -38 & -46 & 42 & 9.59 & 7.01 \\
\hline & & & & -40 & -42 & 42 & 8.46 & 6.48 \\
\hline & & & & -34 & -52 & 40 & 8.00 & 6.25 \\
\hline & & & & -40 & -46 & 50 & 6.55 & 5.45 \\
\hline & & & $\mathrm{R}$ inferior parietal lobule & 38 & -64 & 40 & 8.64 & 6.57 \\
\hline & & & & 36 & -68 & 42 & 8.55 & 6.53 \\
\hline & & & & 36 & -64 & 46 & 8.06 & 6.28 \\
\hline & & & & 44 & -48 & 54 & 7.77 & 6.13 \\
\hline
\end{tabular}


Table A1 | Continued

\begin{tabular}{|c|c|c|c|c|c|c|c|c|}
\hline Condition & $p_{\text {FWE }}$ & $\kappa$ & Region & \multicolumn{3}{|c|}{ Coord. } & $\begin{array}{c}\boldsymbol{T} \text { value } \\
7.70\end{array}$ & $\begin{array}{l}Z \text { value } \\
6.10\end{array}$ \\
\hline & & & & 48 & -46 & 56 & 7.65 & 6.07 \\
\hline & & & L superior parietal lobule & -22 & -70 & 56 & 9.23 & 6.85 \\
\hline & & & & -16 & -68 & 58 & 9.12 & 6.80 \\
\hline & & & & -12 & -72 & 56 & 9.05 & 6.77 \\
\hline & & & R superior parietal lobule & 32 & -54 & 42 & 6.98 & 5.70 \\
\hline & & & & 30 & -64 & 56 & 6.52 & 5.43 \\
\hline & & & & 22 & -72 & 58 & 6.33 & 5.31 \\
\hline & & & L supramarginal gyrus & -35 & -48 & 38 & 9.34 & 6.90 \\
\hline & & & R supramarginal gyrus & 38 & -48 & 38 & 8.36 & 6.43 \\
\hline & & & & 0 & -50 & 60 & 7.07 & 5.75 \\
\hline & & & & -26 & -72 & 40 & 6.85 & 5.62 \\
\hline & & & & -30 & -70 & 42 & 6.37 & 5.34 \\
\hline & & & $\mathrm{R}$ precuneus & 30 & -68 & 42 & 8.52 & 6.51 \\
\hline & & & & 30 & -64 & 40 & 8.51 & 6.51 \\
\hline & & & & 8 & -66 & 58 & 7.74 & 6.12 \\
\hline & & & & 12 & -74 & 54 & 7.70 & 6.10 \\
\hline & & & & 34 & -76 & 38 & 7.17 & 5.81 \\
\hline
\end{tabular}

Tables show significant activations at $p_{F W E}=0.05 . \mathrm{N}=23$ patients and 23 controls, except cortisol with 12 patients and 15 controls. $R$, right; $L$, left; $K$, cluster size. 